\title{
Solute-Atom Segregation/Structure Relations at High-Angle (002) Twist Boundaries in Dilute Ni-Pt Alloys
}

\author{
D. UDLER AND D.N. SEIDMAN \\ Department of Materials Science and Engineering and Materials Research Center, R.R. McCormick School of \\ Engineering and Applied Science, Northwestern University, Evanston, IL 60208-3108
}

Received March 5, 1994; Revised August 23, 1994

\begin{abstract}
Monte Carlo simulations, utilizing embedded atom method (EAM) potentials, are employed to investigate in detail solute-atom segregation behavior at high-angle symmetrical (002) twist boundaries, at $T=850 \mathrm{~K}$, in Pt-3 at.\% Ni and Ni-3 at.\% Pt alloys. Solute enhancement in those alloys occurs on both sides of the phase diagram, although it is considerably higher on the Ni-rich side. The distributions of solute concentrations within the first and the second planes are very inhomogeneous, with the sites highly enhanced in solute being in the minority. The remaining sites exhibit little or no enhancement. The highest level of solute concentrations at individual sites continues to increase with the value of the rotation angle, $\theta$, until saturation occurs at about the $\Sigma=5$ misorientation. The large differences in concentrations between different types of sites suggest the possibility of an ordered grain-boundary phase. The correlation between the structure and solute species concentrations in most cases follows the trends observed for low-angle boundaries: Pt as a solute prefers the structural units of the perfect crystal type, while $\mathrm{Ni}$ as a solute tends to segregate at the filler units associated with the cores of the primary grain boundary dislocations. A strong correlation is observed between the position of a site in the first or second (002) plane and the plane of the interface. Rigid-body translations are detected for two boundaries on the Pt-rich side of the phase diagram. Roughening and possible structural multiplicity occur in the $\Sigma=5$ boundary on the Ni-rich side. The same boundary on the Pt-rich side of the phase diagram exhibits a considerable amount of structural and chemical disorder.
\end{abstract}

Keywords: grain boundaries, solute-atom concentration, Monte Carlo simulations, nickel-platinum alloys

\section{Introduction}

Solute-atom segregation is the change of local composition of an alloy near an imperfection. This phenomenon is often observed at grain boundaries (GBs) and is of great theoretical and practical importance [1-5]. The phase space in which GB solute-atom segregation occurs is that of GB structure; the five macroscopic geometrical degrees of freedom (DOFs), plus the conventional state variables-temperature, pressure and bulk composition-are the relevant thermodynamic state variables [6]. For a single-phase alloy this phase space has $6+C$ dimensions, where $C$ is the number of chemical components in the alloy. The three so-called microscopic degrees of freedom, that is, the rigid-body translation vector between two grains, are assumed to be fully relaxed to minimize the Gibbs free energy of a bicrystal. In our previous papers we focused mainly on the regularities of solute-atom segregation at low-angle (002) twist boundaries in $\mathrm{Au}-$ Pt [7-11] and Ni-Pt [10, 12] dilute alloys. And we identified segregation patterns at those boundaries and showed the importance of interactions between solute atoms and the elastic stress fields of GBs.

An analysis of GB segregation normally starts with the fundamental relation between the Gibbsian interfacial excess of $\Gamma_{\text {solute }}$, and the thermodynamic driving force-the reduction of the interfacial free energy, $\gamma$, of a GB:

$$
\Gamma_{\text {solute }}=-\left(\frac{\partial \gamma}{\partial \mu^{\prime}}\right)_{T, P, \text { specific geomerrical DOFs }},
$$

where $\mu^{\prime}$ is the chemical potential of a solute atom. The next step involves simple phenomenological models, inspired by the classical Langmuir adsorption isotherm for gas adsorption at free surfaces. These models-the Langmuir-McLean isotherm and 
its further modifications (see references [13-18] and references therein)-are unable to provide physical insight into the mechanisms of solute-atom segregation. On the contrary, they are aimed at interpreting experimental data based on assumed atomic mechanisms. The thermodynamics of GB solid solutions is postulated-not derived. At present a powerful method capable of dealing with these issues and obtaining detailed information about the physical mechanisms of segregation is computer simulations utilizing realistic interatomic potentials.

Another important motivation for computer simulations is the following. It is common knowledge that GB solute-atom segregation can alter significantlyeven drastically-practically all important properties of GBs: interfacial free energy, cohesion, mobility, etc. Specific mechanisms for such property changes are still poorly understood. Traditionally, correlations are sought between those changes and integral quanti-

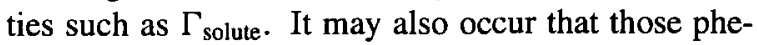
nomena are sensitive to the distribution of segregated solute atoms near an interface and the binding energies involved, not just $\Gamma_{\text {solute }}$. For example, in the phenomenological treatments of GB solute drag [19-22] an important parameter is the "interaction potential" between a boundary and a solute atom. The pre-fracture and fracture behavior at GBs is sensitive to the screening of external stress fields by local stresses [23-24]; those local stresses may be relieved by solute-atom segregation to particular sites.

At low-angle (002) twist boundaries in cubic metals atomic relaxations lead to the formation of a square grid of localized orthogonal screw dislocations. The resulting twist boundary is in a state of pure shear with no long range stress field $[25,26]$. This description appears to be an excellent one for twist boundaries in metals [27]. The screw dislocations are the so-called primary grain boundary dislocations (PGBDs) whose Burgers vectors $(b)$ are $(a / 2)\langle 110\rangle$-type ( $a$ is the lattice constant) in the face-centered cubic lattice. The atomic displacements within the square cells of the PGBDs are rotations around the elements of Bollmann's O-lattice. The spacing of the grid is given by the Read-Shockley relation:

$$
d=\frac{|\mathbf{b}|}{2 \sin (\theta / 2)}=\frac{a / \sqrt{2}}{2 \sin (\theta / 2)} .
$$

The deviation from an exact coincident site lattice (CSL) orientation produces a grid of secondary grain boundary dislocations (SGBDs) with the spacing given by the same Read-Shockley relation, with $\theta$ replaced by
$|\Delta \theta|$; where $\Delta \theta$ is the deviation angle measured from the exact CSL orientation [28]. The intersections of the PGBD lines form patches of the $\Sigma=5$ structure. The localization of the misfit falls off with distance from the interface, and the dislocation cores become broader and less pronounced. At sufficiently large angles $\left(\theta \geq 22.6^{\circ}\right)$ the areas of the regions of "good" atomic fit become comparable to those of the intersection regions, and more complicated high-angle twist boundaries appear that can be described by a structural unit (SU) model $[29,30]$. It is postulated that every GB structure can be decomposed into discrete small patterns of atomic sites, the so-called SUs. The SUs are periodic cells of the boundaries with the lowest periodicity - the so-called favored boundaries. For the (002) twist boundaries the following SUs have been identified:

(1) Perfect crystal units, bounded by $(1 / 2)\langle 110\rangle$ and $(1 / 2)\langle 100\rangle$-type vectors and denoted $\mathrm{A}$ and $\alpha$ respectively;

(2) "Filler" units that make up the cores of the PGBDs; and

(3) CSL $\Sigma=5$ units, bounded by $(1 / 2)\langle 210\rangle$ and $(1 / 2)\langle 310\rangle$ vectors and denoted $B$ and $\beta$ respectively.

For twist boundaries with misorientations $\theta<22.6^{\circ}$ only the $A$ and $\beta$ units occur. In the angular range between $22.6^{\circ}$ and $36.9^{\circ}$ two alternative decompositions are possible $(A, \beta)$ and $(\alpha, \beta)$; while for $\theta>36.9^{\circ}$ only the $B$ and $\beta$ units occur. Filler units may appear throughout the misorientation range to accommodate the residual mismatch. At the intersections of the PGBDs the $B$ and $\beta$ units always occur.

It is emphasized that the bulk of our current knowledge about the structure of high-angle boundaries and the SUs involved has been inferred primarily from lattice statics simulations performed at $0 \mathrm{~K}$ [29-34] and $x$-ray diffraction studies at room temperature [32]. Those studies demonstrate, in some cases [31], the existence of alternative GB structures with energies close to those of the CSL structures. There are only a few finite-temperature studies [32, 35-44], and hence we know very little about so-called structural multiplicity ${ }^{1}$ and possible phase transitions and phase equilibria at GBs [45]. Recently, deterministic free-energy minimization studies, in the local-harmonic approximation, revealed a phase transition from the CSL to type I structure in a $\Sigma=5$ high-angle twist boundary in gold at about $315 \mathrm{~K}$ [40], as well as in some other (002) twist boundaries in gold [39]. 
In our previous papers [7] Monte Carlo simulations were performed for the type I and type II as well as the CSL $\Sigma=5$ high-angle boundaries in a Pt-1 at. $\% \mathrm{Au}$ alloy at $850 \mathrm{~K}$. The type I structure reverted to the CSL structure and the type II structure remained metastable. In the present paper we report some observations of structural multiplicity at high-angle grain boundaries at $850 \mathrm{~K}$. A systematic investigation of these effects is in progress [46]. We have found [7-12] regularities relating solute-atom segregation to the structure of (002) twist boundaries and showed the importance of interactions between solute atoms and the elastic stress fields of twist boundaries.

In the present paper we investigate in detail soluteatom segregation at high-angle (002) boundaries in the Ni-Pt system. The main questions we address are:

- What is the relation between solute-atom segregation and the atomic structure of high-angle boundaries?

- What is the distribution of solute-atom concentrations in different SUs and individual atomic sites?

- Is there any dependence between the misorientation angle and the values of solute-atom concentrations?

- What is the difference between the special and general GBs with respect to solute-atom segregation?

\section{Monte Carlo Simulations}

To study equilibrium solute-atom segregation at GBs at elevated temperatures we employ Monte Carlo simulations in the transmutational ensemble. The computational method is described in detail in reference [47] and was used in a number of investigations [4, 7$12,48]$. We briefly recapitulate the principal aspects of the technique. During the simulation, the temperature, the total number of particles and the difference in the excess chemical potentials are held constant. The total volume of the computational cell is allowed to relax, with the net effect being a constant pressure in the ensemble. The chemical identities of particles are changed, so that the atomic fraction of each component is a variable. The chemical composition in the reference bulk region is thus maintained constant, due to the constant difference of excess chemical potentials, while near the imperfections it changes to its correct equilibrium value. We utilize the fundamental algorithm of Metropolis et al. [49] to accept an attempted change. At each Monte Carlo step an atom is chosen at random. Then the following sequence of attempts is performed:
1. The chemical identity of an atom is changed; this step is for modifying the local composition of an alloy, and it is basic to the phenomenon of soluteatom segregation.

2. The displacement of an atom from its position by a random vector with a magnitude smaller then $0.01 \mathrm{~nm}$; this step allows for relaxations on atomic scale. Note well that steps 1 and 2 are made simultaneously.

3. After, on the average, all the atoms in the bicrystal have undergone the above two attempts an overall volume relaxation is implemented that involves a change in one or more periodic lengths and a corresponding rescaling of coordinates of all the atoms in the bicrystal by a small random quantity. This procedure changes the volume of the computational cell to maintain the bicrystal at constant pressure.

4. A relative translation of the two grains in the plane of the interface by a random vector is attempted with the same time periodicity as step 3 . It allows the complete relaxation of all microscopic degrees of freedom of a GB. Thus, steps $2,3,4$ represent sampling of the $3 \mathrm{~N}$-dimensional configurational space of the given bicrystal. Note well, that the Metropolis algorithm guarantees convergence to equilibrium and correct weighting for averaging independent of the exact implementation of the trial moves.

The total value of the internal energy, $E_{\text {tot }}$, is calculated using embedded atom method (EAM) potentials [50-53], that have been developed for six face-centered cubic elements ( $\mathrm{Ag}, \mathrm{Au}, \mathrm{Cu}, \mathrm{Ni}, \mathrm{Pd}$ and $\mathrm{Pt}$ ). The EAM potentials are empirical many-body continuous potentials that have been used extensively for studying different physical problems in materials science [54]. We employed the so-called universal version of the EAM potentials [52] which are not specifically fitted to dilute heats of solution, but reproduce the experimental values.

Prior to simulations for GBs, similar simulations are run for a perfect single crystal (4000 atoms) to determine the value of $\Delta \mu$-the difference in the excess chemical potentials--corresponding to the requisite value of solute-atom concentration. The equilibrium lattice constants for given concentrations are also obtained from those runs $\left(10^{4}\right.$ Monte Carlo steps per atom). In the present study we consider dilute ( 3 at.\% solute) alloys on both sides of the phase diagram, and we are also currently working on GB segregation in the ordered $\mathrm{Ni}_{3} \mathrm{Pt}$ alloy. An effort is now under way to map out the entire bulk phase diagram for the Ni-Pt EAM potential. Besides the solid solution regions we have 
reliably identified the presence of the ordered phases $\mathrm{Ni}_{3} \mathrm{Pt}$ and $\mathrm{NiPt}$ in a wide temperature range.

The computational cell has three-dimensional periodic boundary conditions and contains two grains rotated about an [001] direction to a given twist angle $\theta$. This angle is the only macroscopic geometric degree of freedom varied in this investigation. Due to the fourfold symmetry of the interface (point group symmetry $4 \mathrm{~mm}$ ) the irreducible interval of the rotation angle is $\theta=0^{\circ}$ to $45^{\circ}$. Physically, there are two crystallographically identical, but physically different GBs in a bicrystal. The separation between two interfaces is taken to be $16(002)$ plane, that is, each grain consists of 16 planes. We previously found this separation to be sufficient to avoid elastic interactions between the GBs and to provide a region of unstressed perfect crystalthe bulk-in the middle of each grain. In our Monte Carlo simulations we use a computational cell that is approximately (4 to 5$) \mathrm{nm} \times(4$ to 5$) \mathrm{nm}$ in the plane of the interface-this corresponds to between 200 to 300 atoms in one atomic plane-and is about $6.3 \mathrm{~nm}$ in the direction normal to the (002) GB plane. Thus, the overall number of atoms in the system varies between 6400 to 9600 . The first $2.5 \times 10^{3}$ Monte Carlo steps per atom of each run is used for equilibration. The averaging is performed for the next $3 \times 10^{3}$ or $10^{4}$ Monte Carlo steps per atom, depending on the GB. The time step for averaging was 10 Monte Carlo steps per atom to avoid temporal correlation effects. The averaging is performed over the coordinates of each atom $i$, and this average is identified with the position of an atomic site $i$. In the solid state each atom is expected to stay at a particular atomic site, deviating only to the distances of the order of vibrational amplitudes, that are much smaller than the shortest distance between atomic sites. During the course of a simulation the chemical identity of each atom is changed, so the fraction of time an atom spends as a given species $i$ is taken to be the average concentration of this species at an atomic site $i$. These terms have a clear physical meaning and are used to discuss the phenomenon of solute-atom segregation.

To avoid the effects of the interfacial tension of GBs-which may be considerable in a small computational system-we fix the area of an interface at the bulk value, for a given temperature and bulk concentration of solute, by not permitting relaxation of the two periodic lengths in the plane of an interface. The periodic length in the direction normal to the interface is allowed to relax to relieve stresses in the bulk. This procedure yields the same results as when a larger bicrystal ( 30 planes between the GBs) is employed that does not fix the area of an interface. Near an interface the atomic relaxations produce an expansion (volume excess) analogous to that observed near GBs in real materials [55-57]. The bicrystals employed are ones where the [001] direction is perpendicular to the (002) interface plane; the geometric interface lies between two (002) planes and it contains no atoms. The highangle boundaries studied correspond to the following $\theta$ misorientations: $16.3^{\circ}(\Sigma=25), 22.6^{\circ}(\Sigma=13)$, $28.1^{\circ}(\Sigma=17), 33.9^{\circ}(\Sigma=289), 36.9^{\circ}(\Sigma=5)$, $41.1^{\circ}(\Sigma=73)$ and $43.6^{\circ}(\Sigma=29)$.

\section{Solute-Atom Segregation Behavior}

\subsection{Distribution of Concentrations at Atomic Sites}

Figures 1 and 2 exhibit the distributions of solute-atom concentrations for the first two (002) planes adjacent to the interface in all seven high-angle GBs on both sides of the phase diagram. The plots represent solute concentrations at individual atomic sites in a plane, with each point corresponding to one atomic site. The sites are arranged in descending order of solute concentration. The number of sites in a plane varies between 208 and 289, depending on the GB. These plots have several important advantages over conventional histograms as they carry more detailed information and are relatively smooth. Histograms require a subtle balance between the bin size and the desired picture- too small a bin size leads to a considerable amount of noise, while too coarse a bin size smoothes out important information. The optimal size of a bin is often different for different data sets. The descending value plots allow one to identify discrete levels of segregation at symmetrically equivalent sites-they correspond to regions of the curve with small slopes. Alternatively, a large slope corresponds to a crossover between two discrete levels. Finally, the average concentration in the plane is simply the area under the curve divided by the extent of the $x$-axis (that is, the total number of sites in the plane).

The following results are gleaned from Figs. 1 and 2.

1. For both sides of the phase diagram solute enhancement occurs both in the 1st and 2nd planes adjacent to the interface. The magnitude of the effect is, however, much greater in the 1 st plane.

2. The distribution of concentrations at atomic sites is very inhomogeneous, ranging in some cases from the bulk concentration of 3 at.\% solute to values 


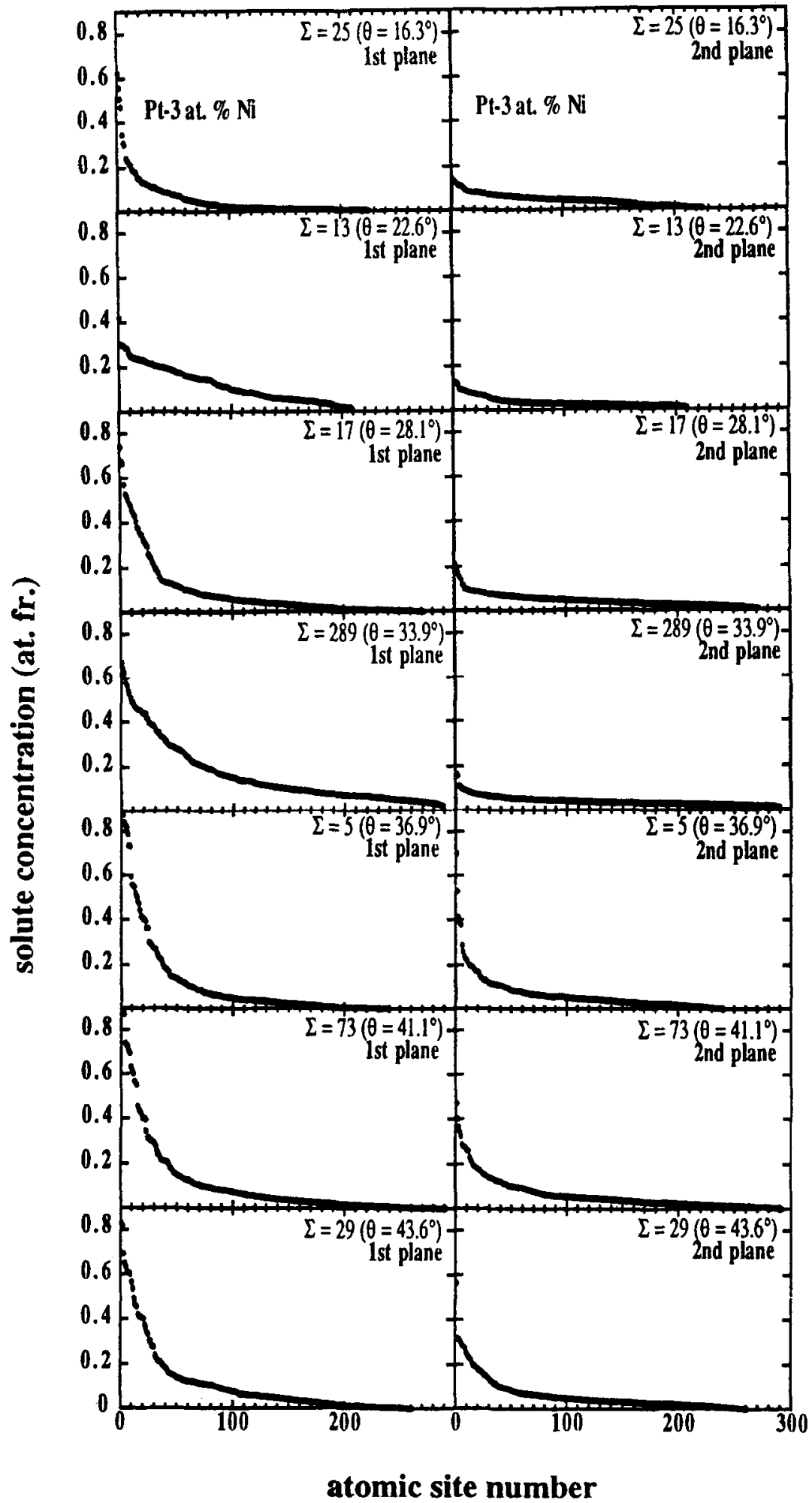

Fig. 1. Descending value plots of solute concentrations at the two first planes adjacent to the interface for (002) twist boundaries in the Pt-3 at. \% $\mathrm{Ni}$ alloy. Each atomic site is represented by a point and the sites are arranged in descending order of concentrations. 


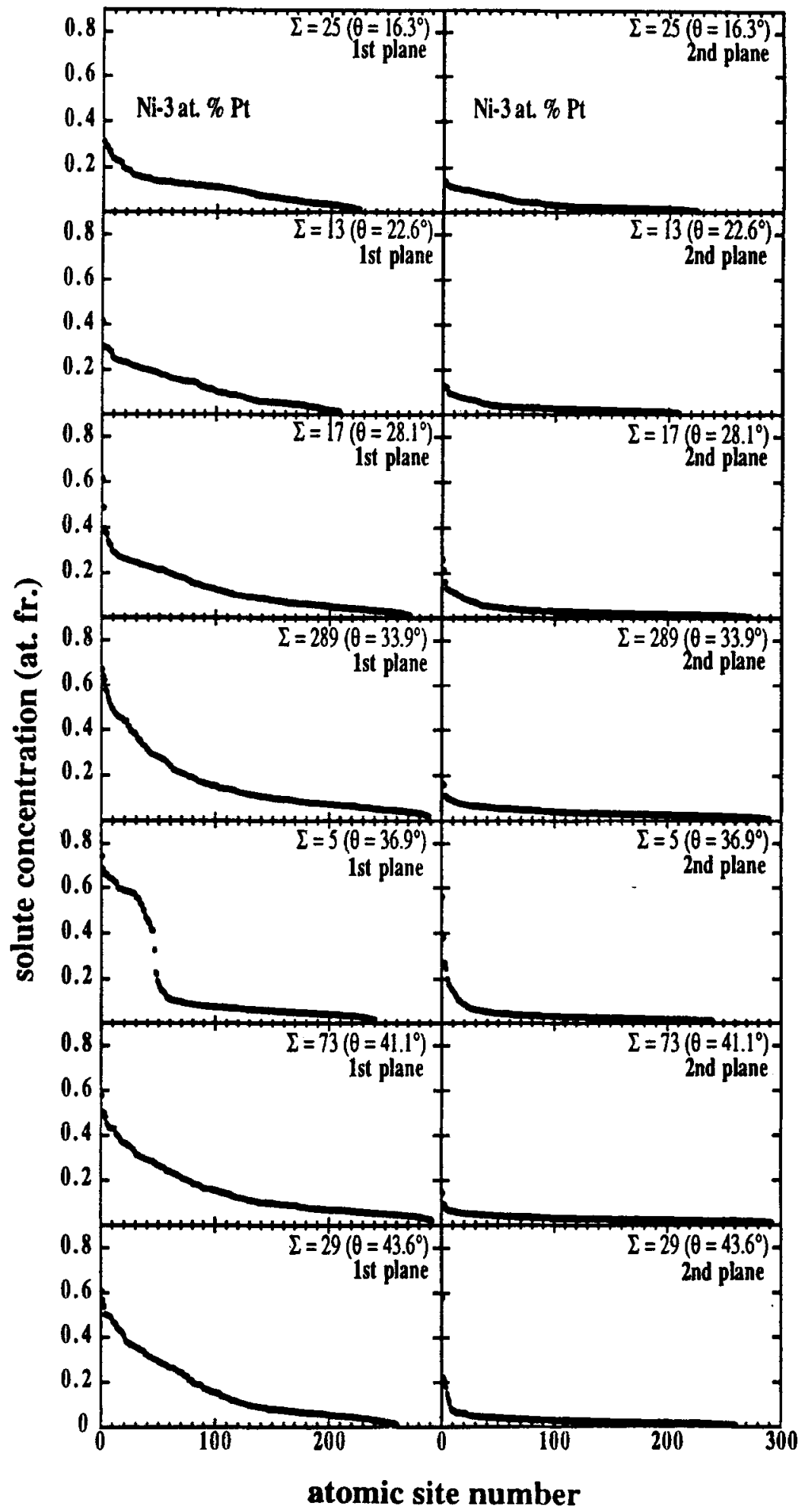

Fig. 2. Same plots as in Fig. 1 for the Ni-3 at.\% Pt alloy. 
that are a factor of 25 greater. On the Pt-rich side (Fig. 1) the tail even falls below 3 at. $\% \mathrm{Ni}$, indicating depletion at those sites. On most occasions the maximum concentration levels of solute are higher on the Ni-rich side (Fig. 2).

3. There is a clear trend for an increase in the maximum concentration levels of solute with increasing $\theta$. Starting, however, with $\Sigma=5$ saturation occurs.

4. The only case where the segregation levels appear to be well separated is in $\Sigma=5$ boundaries on the Ni-rich side of the phase diagram. In all other cases different segregation levels (corresponding to different binding free energies of solute atoms at different types of symmetrically equivalent locations) overlap significantly. Apart from the $\Sigma=5$ case there seems to be no apparent difference in the distributions of concentrations between the special boundaries with small values of $\Sigma$ and general boundaries with $\Sigma$ values greater than 30 . Each boundary, however, appears to have a unique distribution of solute-atom concentrations that is statistically significant.

\subsection{Structural Regularities of Solute-Atom Segregation}

Figures 3 to 14 exhibit the detailed structures of the interfaces and solute-atom concentrations at different atomic sites. The (a) portions of Figs. 3 to 10 and 13,14 exhibit the structures of the interfaces with the microscopic degrees of freedom relaxed (rigidbody translations of the two grains), but without local atomic relaxations near the interface. They are produced by projecting the average atomic positions from four (002), from both sides of the interface, onto the interface; thus information from eight planes is exhibited. Parts (a) of those figures represent an important reference frame for discussion of the interfaces, that are depicted in the (b) portions of the same figures. The plots exhibited in (b) display the average positions of atomic sites in two (002) planes on each side of the interface; thus information from four planes is exhibited. The sites with solute concentrations more than two standard deviations greater than the bulk value (that is, greater than 4.5 at.\%) are shown in black. The remainder are taken to have the bulk concentration of solute and they are depicted by open circles.

3.2.1 The $\Sigma=25\left(\theta=16.3^{\circ}\right)$. This is the lowestangle boundary discussed in this paper and it exhibits a continuation of the patterns observed in low-angle GBs $[11,12]$. On the Pt-rich side (Fig. 3) the sites that can be attributed to dislocation cores and their intersections are enhanced in solute. While on the Ni-rich side (Fig. 4) the situation is complementary, that is, the dislocation core sites are not enhanced in solute, while the good fit regions are. Note well, that the intersections of the dislocation lines have different structures. According to the SU model $[29,30]$ these regions should be patches with a $\Sigma=5$ structure. This observation points to a possible multiplicity of GB structures at elevated temperatures. These effects are discussed in detail in section 3.2.4.

\subsection{2 $\Sigma=13\left(\theta=22.6^{\circ}\right)$. First compare parts (a)} of Figs. 5 and 6. It is clear that on the Pt-rich side a small but noticeable (about $0.005 \mathrm{~nm}$ ) rigid-body translation in the plane of the interface is present and its direction is $\approx[210]$. Within the present computational setup, however, it is impossible to reliably establish the displacement vector(s) or vectors, because the average picture is actually a superposition of different displacements that occurred during the simulation. The effects of rigid-body translations on free energies of GBs at finite temperatures are investigated by the overlapping distribution Monte Carlo technique and presented elsewhere [46]. On the Pt-rich side (Fig. 5) the sites that are preferentially enhanced in $\mathrm{Ni}$ can be identified with the cores of PGBDs and their intersections, while the areas of good fit are not enhanced. The opposite effect occurs on the Ni-rich side of the phase diagram (Fig. 6), although some of the sites in the areas where the dislocations intersect are enhanced. Note also structural multiplicity of those intersection regions (same as for the $\Sigma=25$ boundary) on both sides of the phase diagram. The coincident sites seem to be enhanced on both sides of the phase diagram, although on the Pt-rich side the trend is not absolute.

\subsubsection{The $\Sigma=17\left(\theta=28.1^{\circ}\right)$. The general trends} are the same as in lower angle boundaries, although a considerable amount of noise, both in structure and the distribution of enhanced sites, is observed on both sides of the phase diagram. The coincident sites are not enhanced in solute on the Pt-rich side (Fig. 7) and they are enhanced on the Ni-rich side (Fig. 8).

3.2.4 The $\Sigma=5\left(\theta=36.9^{\circ}\right)$. This is the most special boundary with the simplest structure-the unit cell has one coincident and four equivalent noncoincident sites both in the first and second planes adjacent 


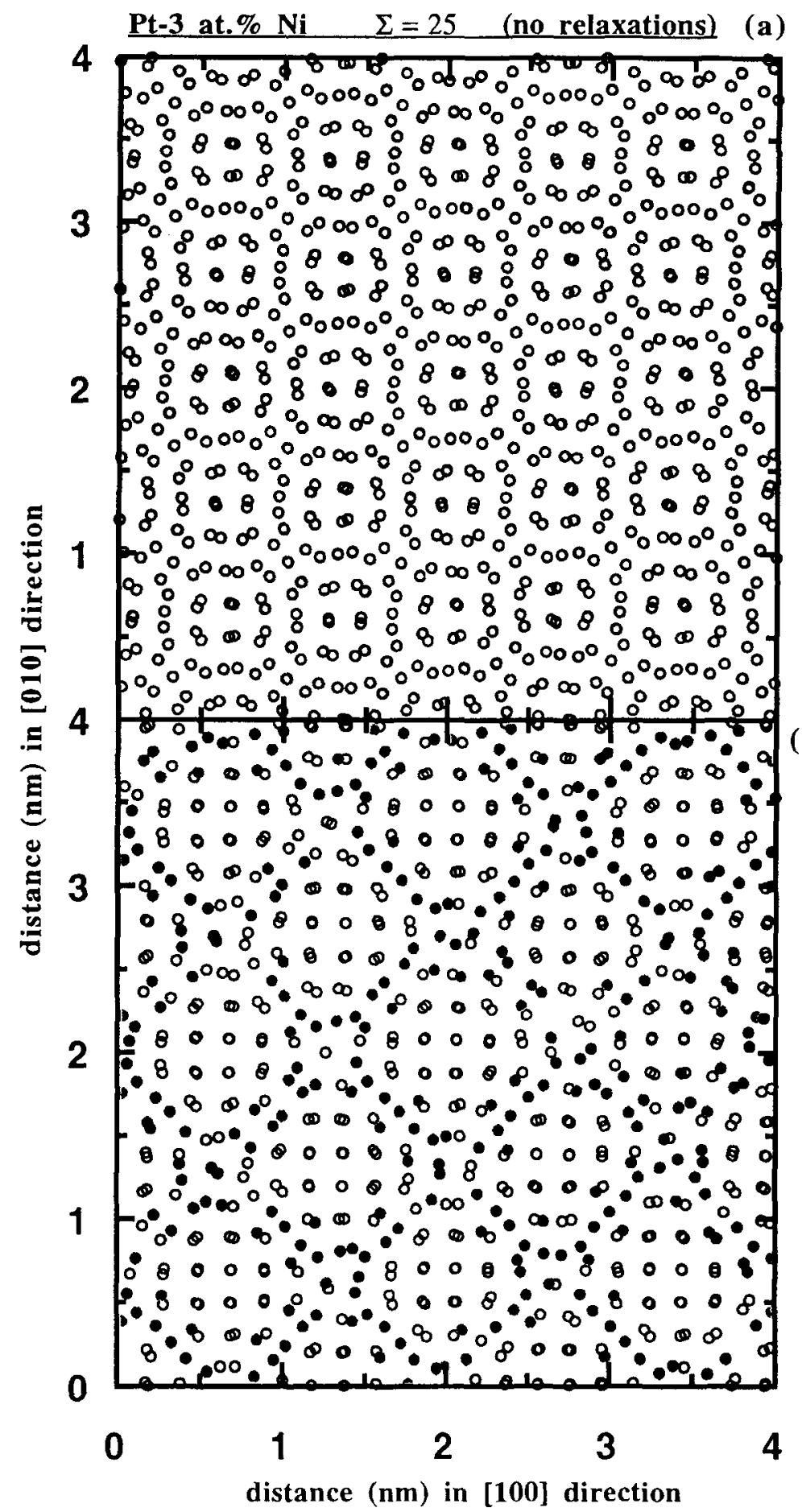

Fig. 3. The structure of and solute-atom segregation at the $\theta=16.3^{\circ}(\Sigma=25)$ boundary in Pt-3 at.\% Ni. The black circles correspond to mean concentrations at atomic sites more than two standard deviations greater than the bulk value (that is, greater than 4.5 at.\% Ni) and the open circles are less than 4.5 at.\% $\mathrm{Ni}$ and are considered to have the bulk concentration. 
Ni-3 at. $\%$ Pt $\quad \Sigma=25$ (no relaxations)

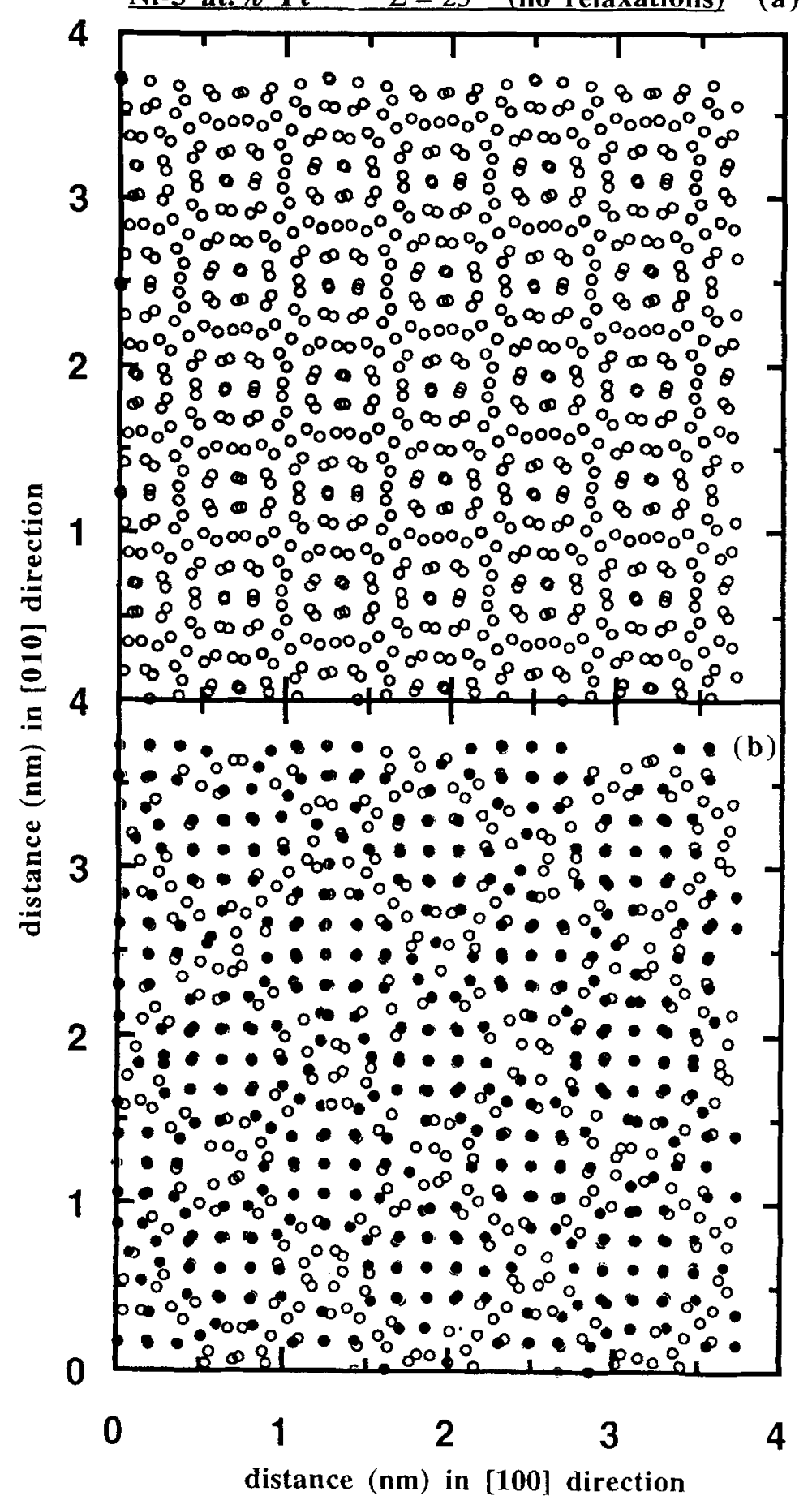

Fig. 4. The structure of and solute-atom segregation at the $\theta=16.3^{\circ}(\Sigma=25)$ boundary in Ni-3 at.\% Pt. The black circles correspond to mean concentrations at atomic sites more than two standard deviations greater than the bulk value (that is, greater than 4.5 at.\% Pt) and the open circles are less than 4.5 at.\% Pt and are considered to have the bulk concentration. 


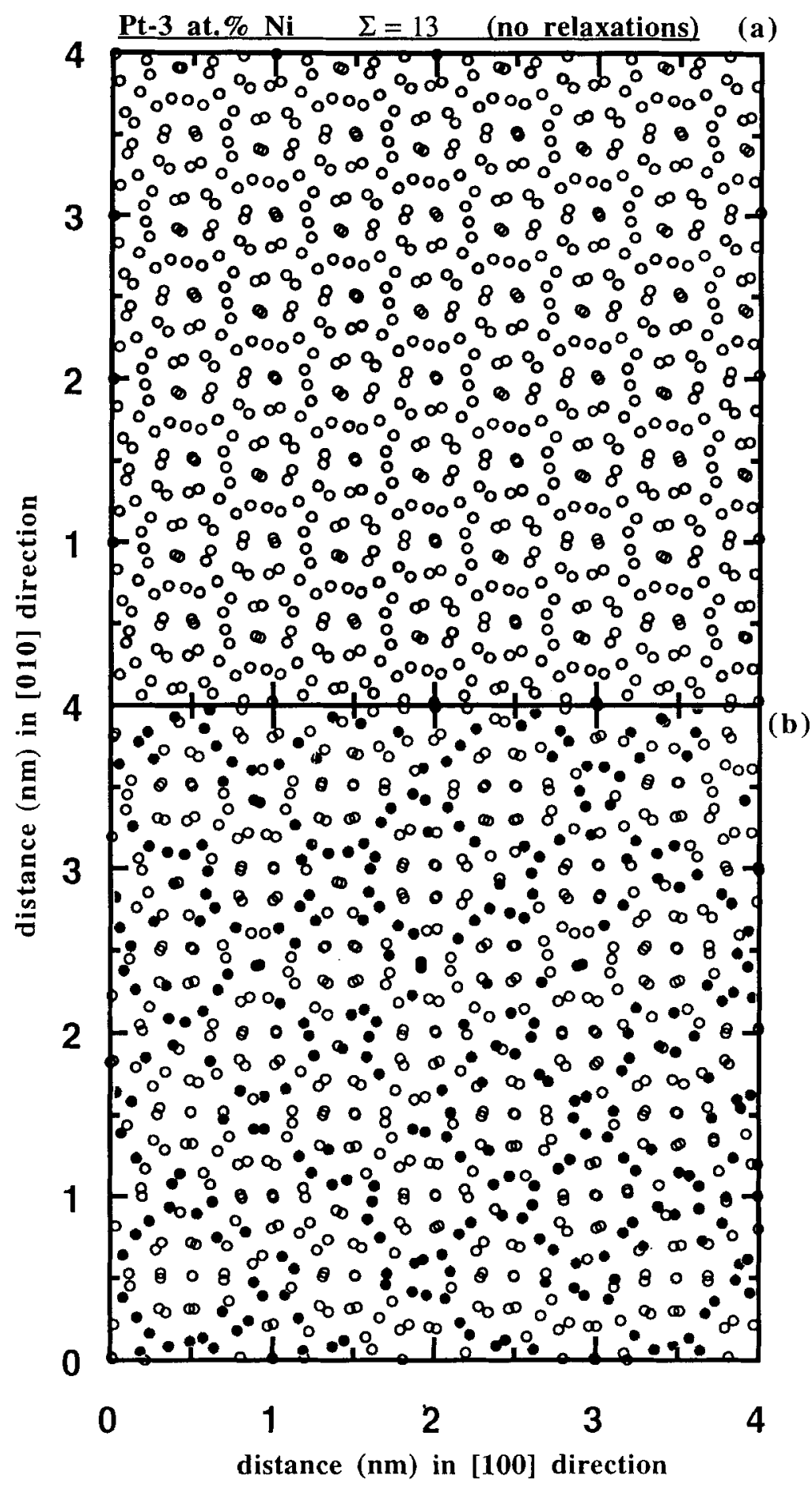

Fig. 5. The structure of and solute-atom segregation at $\theta=22.6^{\circ}(\Sigma=13)$ boundary in Pt-3 at.\% Ni; same nomenclature as in Fig. 3 .

to the interface. First, note a small asymmetry in the unrelaxed pattern in Fig. 9(a), compared to the almost perfect symmetry in Fig. 10(a). It indicates a small (about $0.01 \mathrm{~nm}$ ) rigid-body translation of the two grains in the plane of the interface (in approximately the [310] direction), analogous to that observed in the $\Sigma=13$ boundary. Also note the considerable amount of structural disorder in the GB structure on the Pt-rich side 


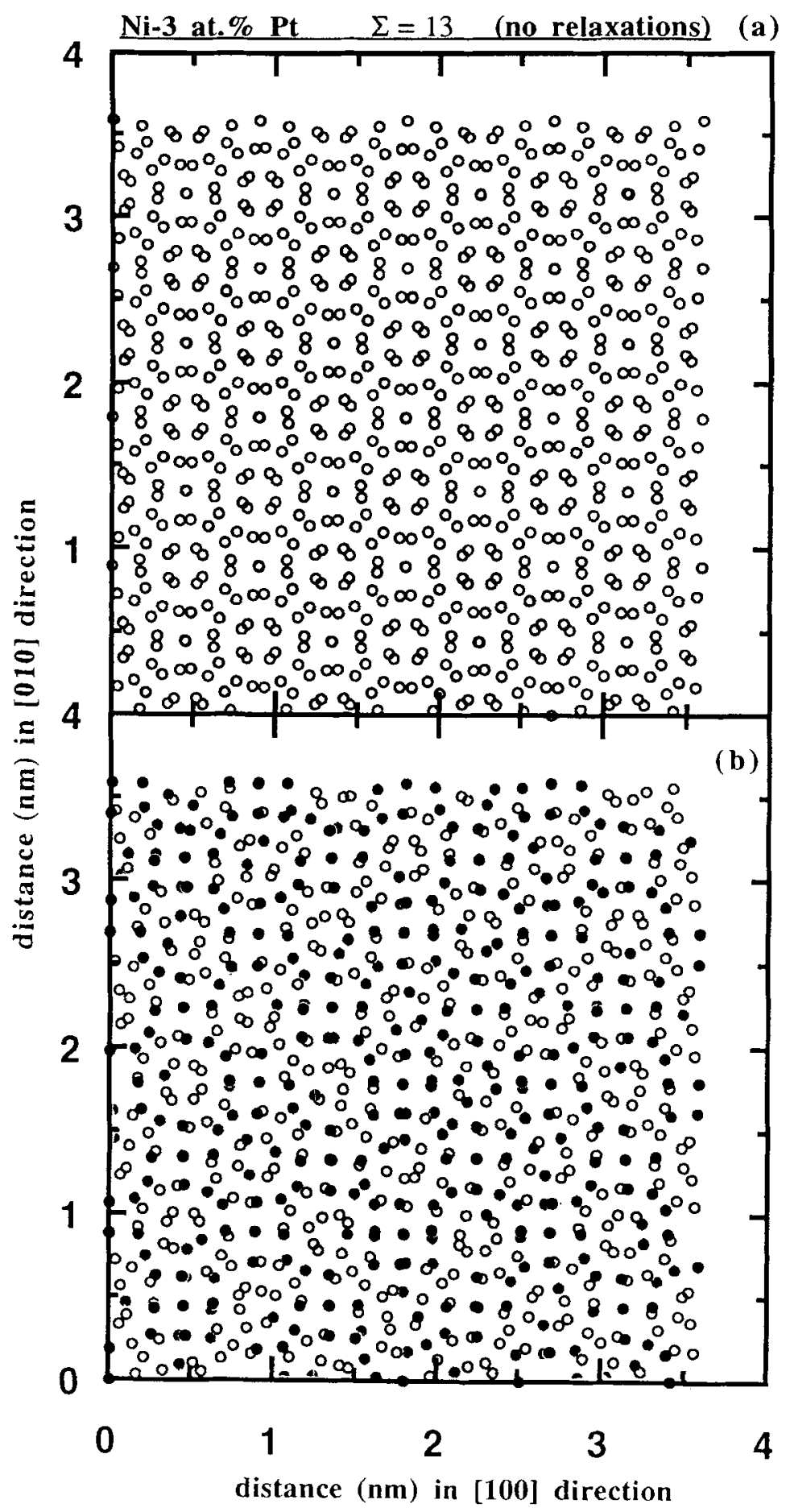

Fig. 6. The structure of and solute-atom segregation at $\theta=22.6^{\circ}(\Sigma=13)$ boundary in Ni-3 at.\% Pt; same nomenclature as in Fig. 4.

of the phase diagram (Fig. 9), compared to a rather regular structure on the $\mathrm{Ni}$-rich side of the phase diagram (Fig. 10). Even this relatively regular structure, however, has a certain concentration of rather uniform imperfections. Their structure is better seen in Fig. 11. Part (a) of this figure exhibits the structure of 


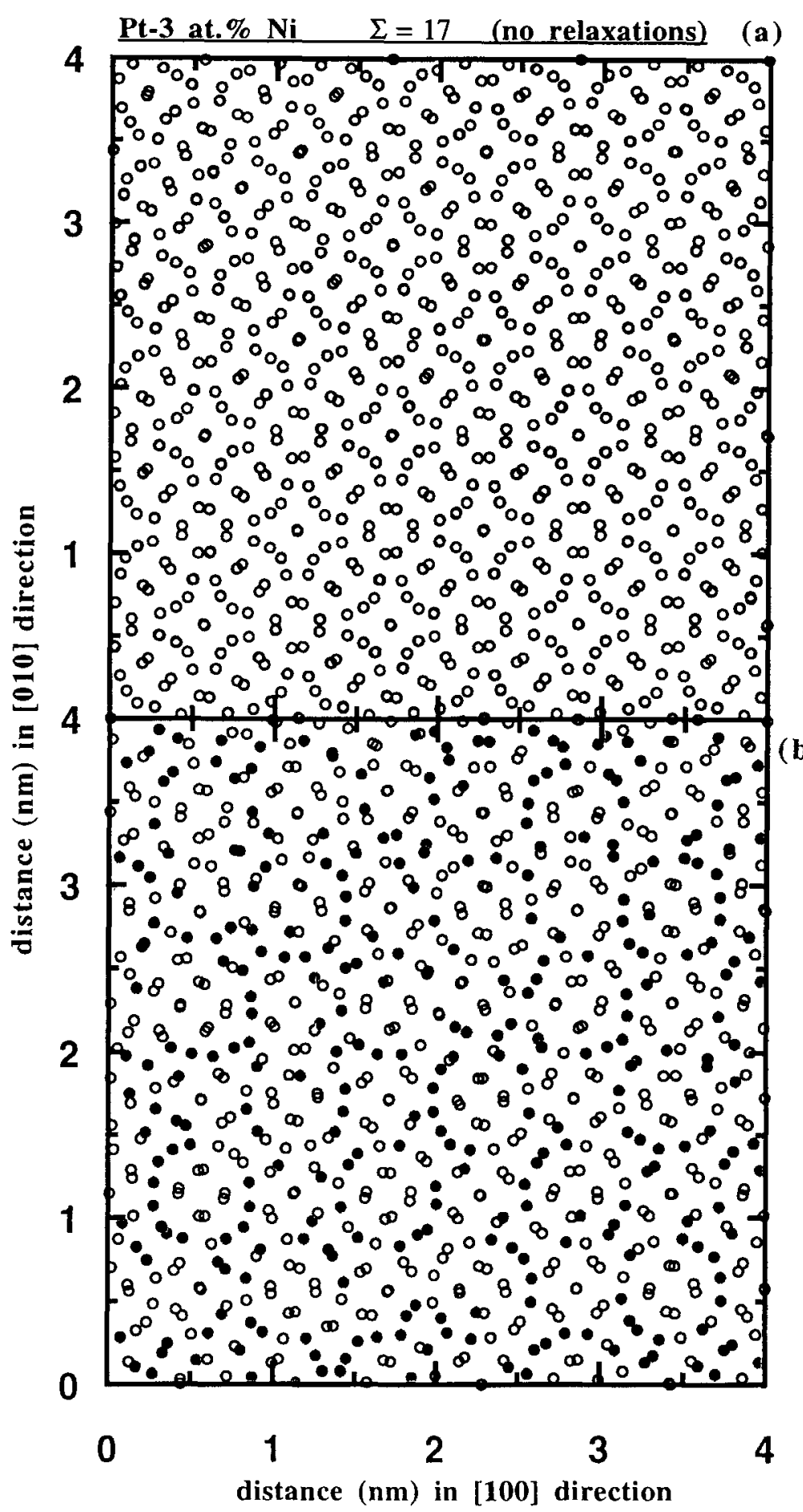

Fig. 7. The structure of and solute-atom segregation at the $\theta=28.1^{\circ}(\Sigma=17)$ boundary in Pt-3 at.\% Ni; same nomenclature as in Fig. 3 .

the two first (002) atomic planes on one side of the interface plane (say, below). Similarly, part (b) shows two atomic planes on the other side of the plane of the interface (say, above). The patches breaking the symmetry of the structure are clearly visible in both plots. They are the $\Sigma=5$ units that spontaneously shift 


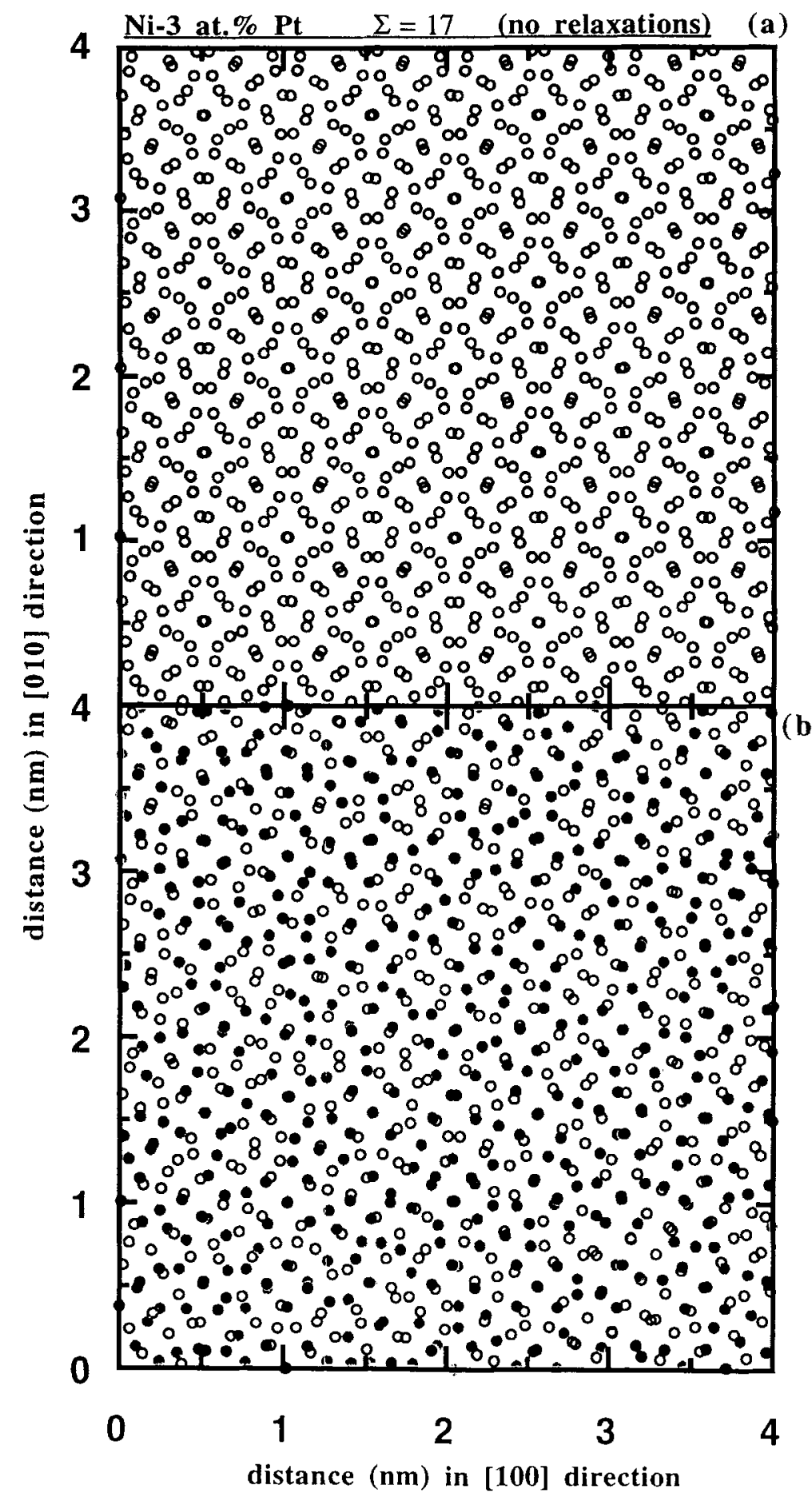

Fig. 8. The structure of and solute-atom segregation at the $\theta=28.1^{\circ}(\Sigma=17)$ boundary in Ni-3 at.\% Pt; same nomenclature as in Fig. 4 .

one atomic plane perpendicular to the interface (above or below). Thus, steps on the GB are formed. A similar phenomenon was observed in molecular dynamics simulations of the $\Sigma=5$ boundary in gold, utilizing an empirical pair potential [35] and the EAM potential for gold [32]. The shifted $\Sigma=5$ units also display a 


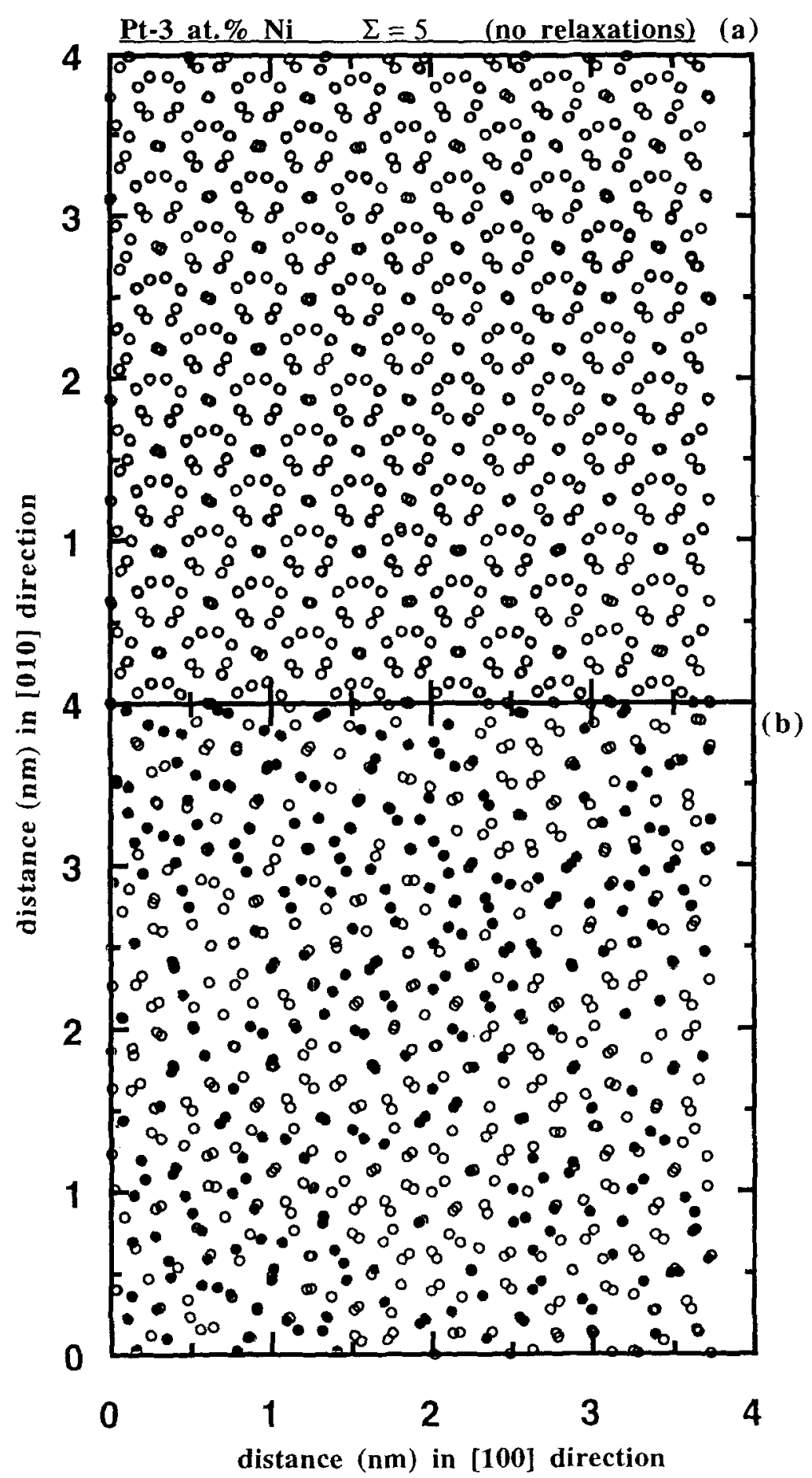

Fig. 9. The structure of and solute-atom segregation at the $\theta=36.9^{\circ}(\Sigma=5)$ boundary in Pt-3 at.\% Ni; same nomenclature as in Fig. 3 .

variety of structures. Two representative shifted units are cut out from Fig. 11 and exhibited as blow-ups in Fig. 12(a) and (b). The structures in parts (a) and (b) of Fig. 12 look significantly different. Both are almost perfectly symmetrical, and it is therefore very unlikely that one of them is a version of the other 


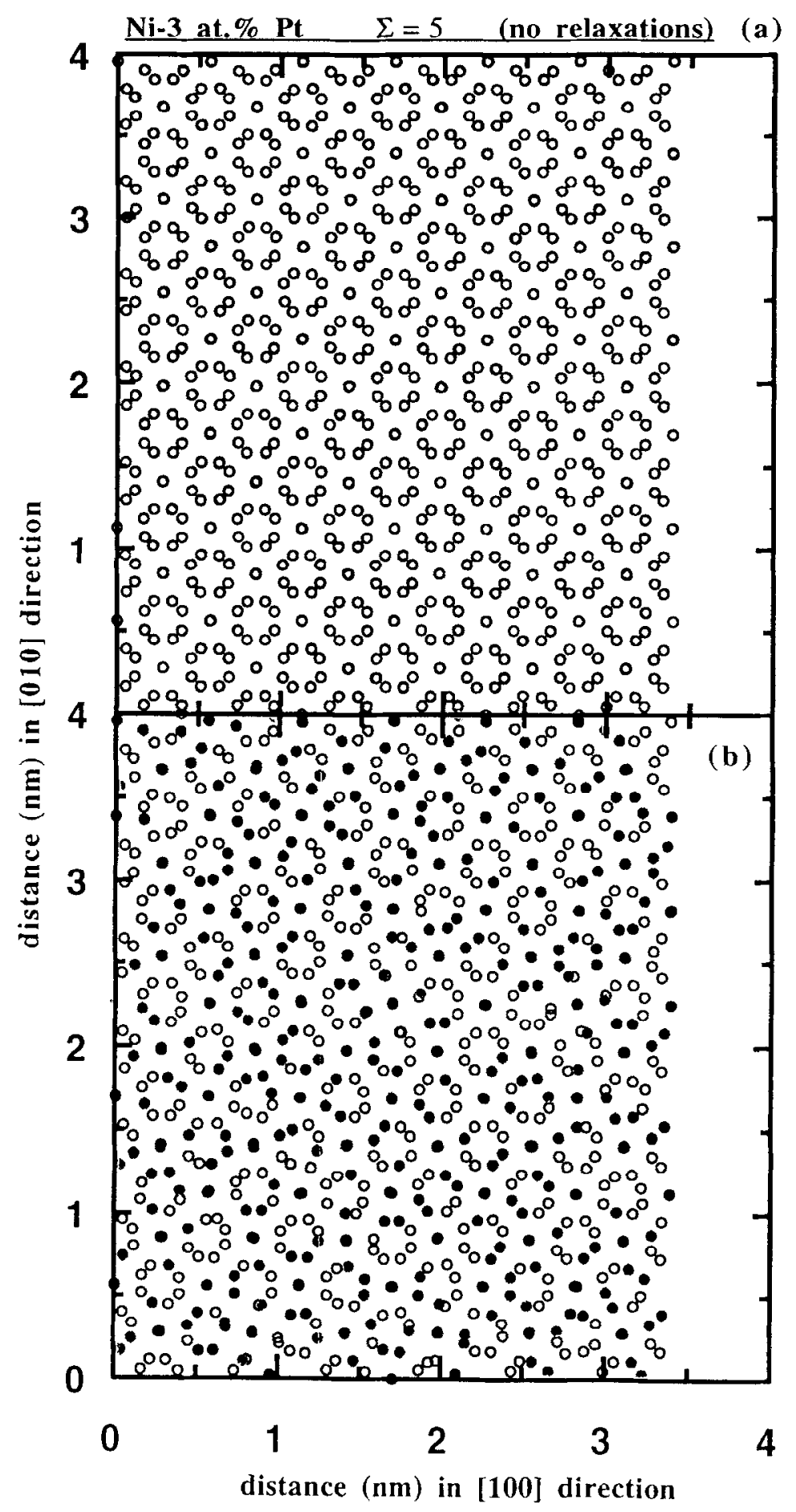

Fig. 10. The structure of and solute-atom segregation at the $\theta=36.9^{\circ}(\Sigma=5)$ boundary in Ni-3 at.\% Pt; same nomenclature as in Fig. 4 .

structure distorted by thermal vibrations-distortions on the scale of thermal vibrations cannot create a new symmetry. It should be borne in mind, however, that the coordinates of the atomic sites are time averages over the whole duration of the simulation. If a fraction of the time was spent in a shifted position, the superpo- 
Ni-3 at. \% Pt $\Sigma=5$

(a)

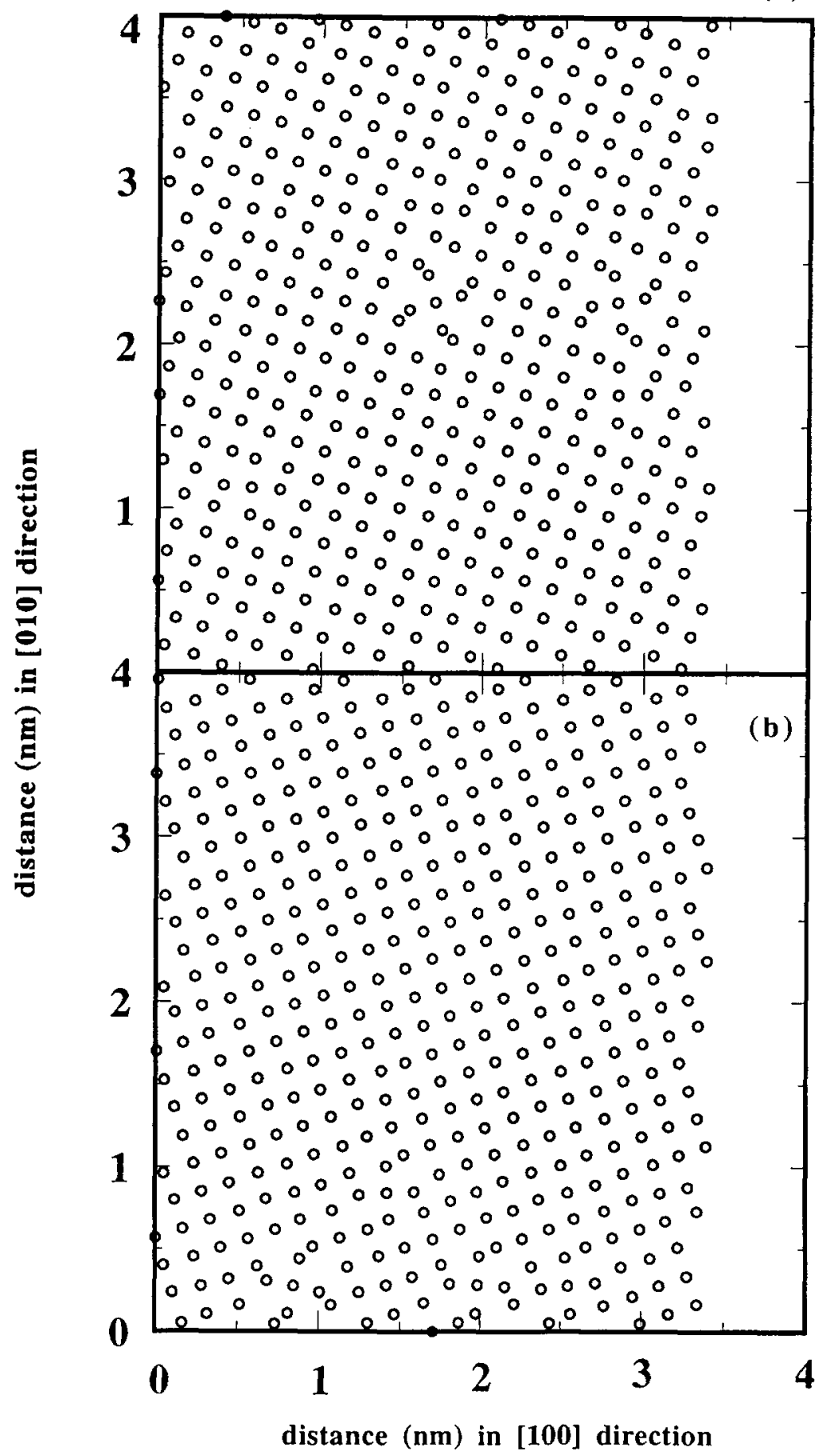

Fig. 11. The relaxed structure of the $\theta=36.9^{\circ}(\Sigma=5)$ boundary in Ni-3 at.\% Pt (same as in Fig. 10(b)). Parts (a) and (b) exhibit pairs of the (002) atomic planes each side of the interface. Unit cells with a structure, different from the majority of the unit cells, are seen in the middle of (a) and in the bottom of (b). These unit cells are actually unit steps, penetrating one (002) interplanar distance to the corresponding side of the main interface plane. 


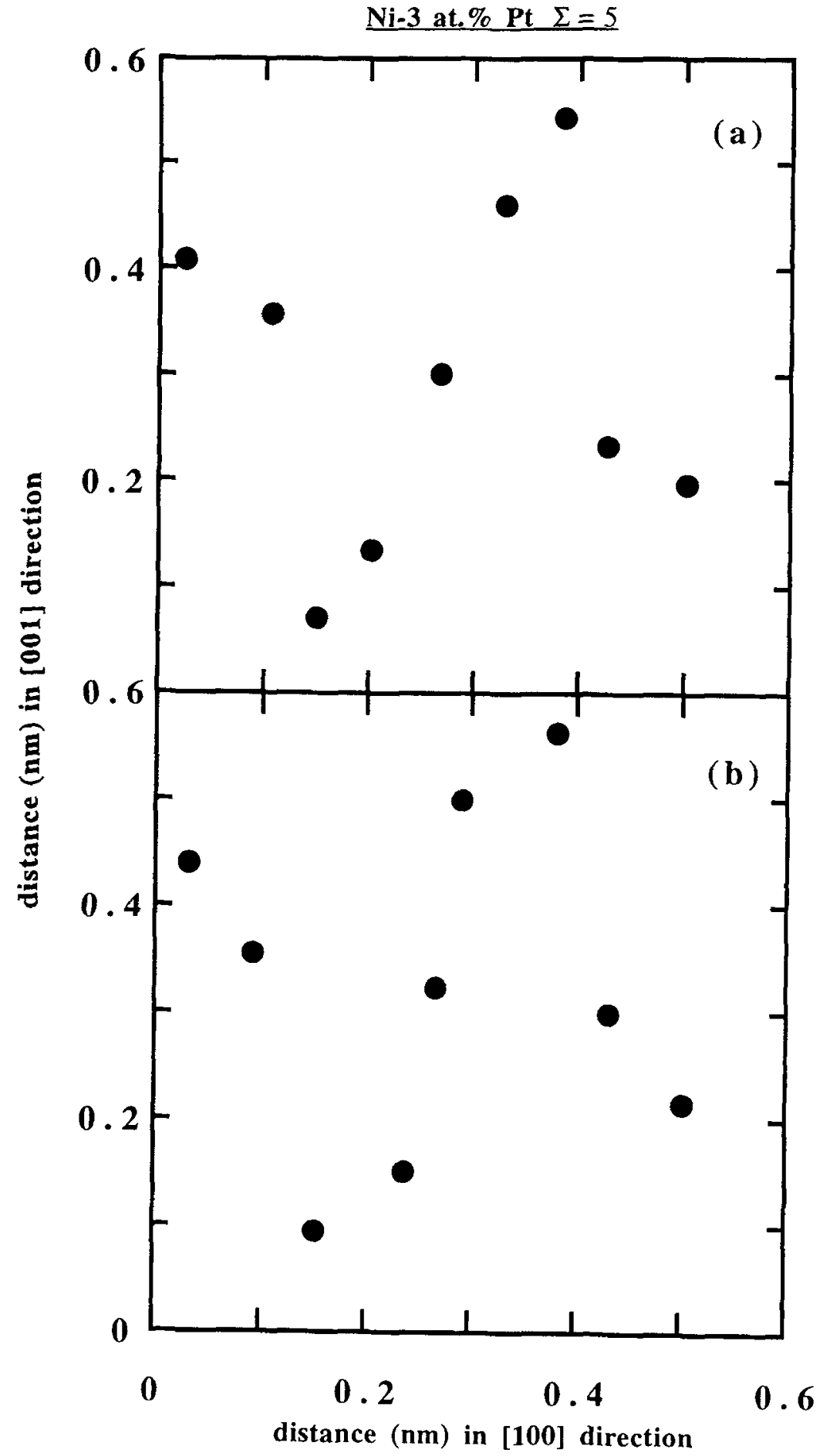

Fig. 12. The parts (a) and (b) are blow-ups of the two step units [from Fig. 11(b)] of the $\theta=36.9^{\circ}(\Sigma=5)$ boundary in the Ni-3 at.\% Pt alloy. For the sake of clarity only the two (002) planes are displayed. Note the difference between the two plots in the positions of the non-coincident atomic sites. 


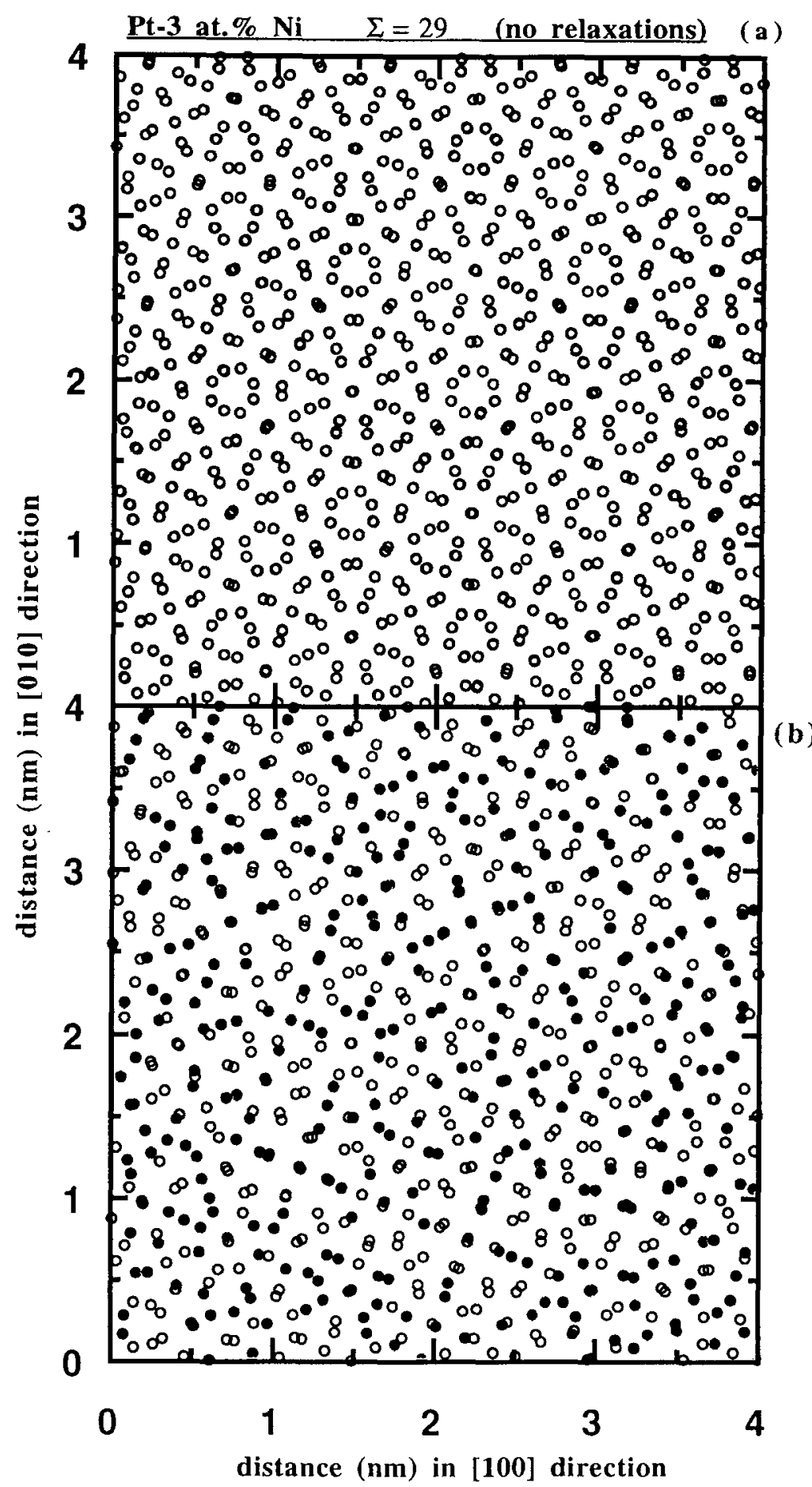

Fig. 13. The structure of and solute-atom segregation at the $\theta=43.6(\Sigma=29)$ boundary in Pt-3 at.\% Ni; same nomenclature as in Fig. 3 .

sition of the structures could give rise to such effects. Alternatively, they may be a manifestation of really diverse structures, as suggested earlier [35]. Finally, we recall that structural changes are observed also at the $\Sigma=5$ patches of the $\Sigma=25$ and $\Sigma=13$ GBs.

With respect to segregation there is a clear trend for 


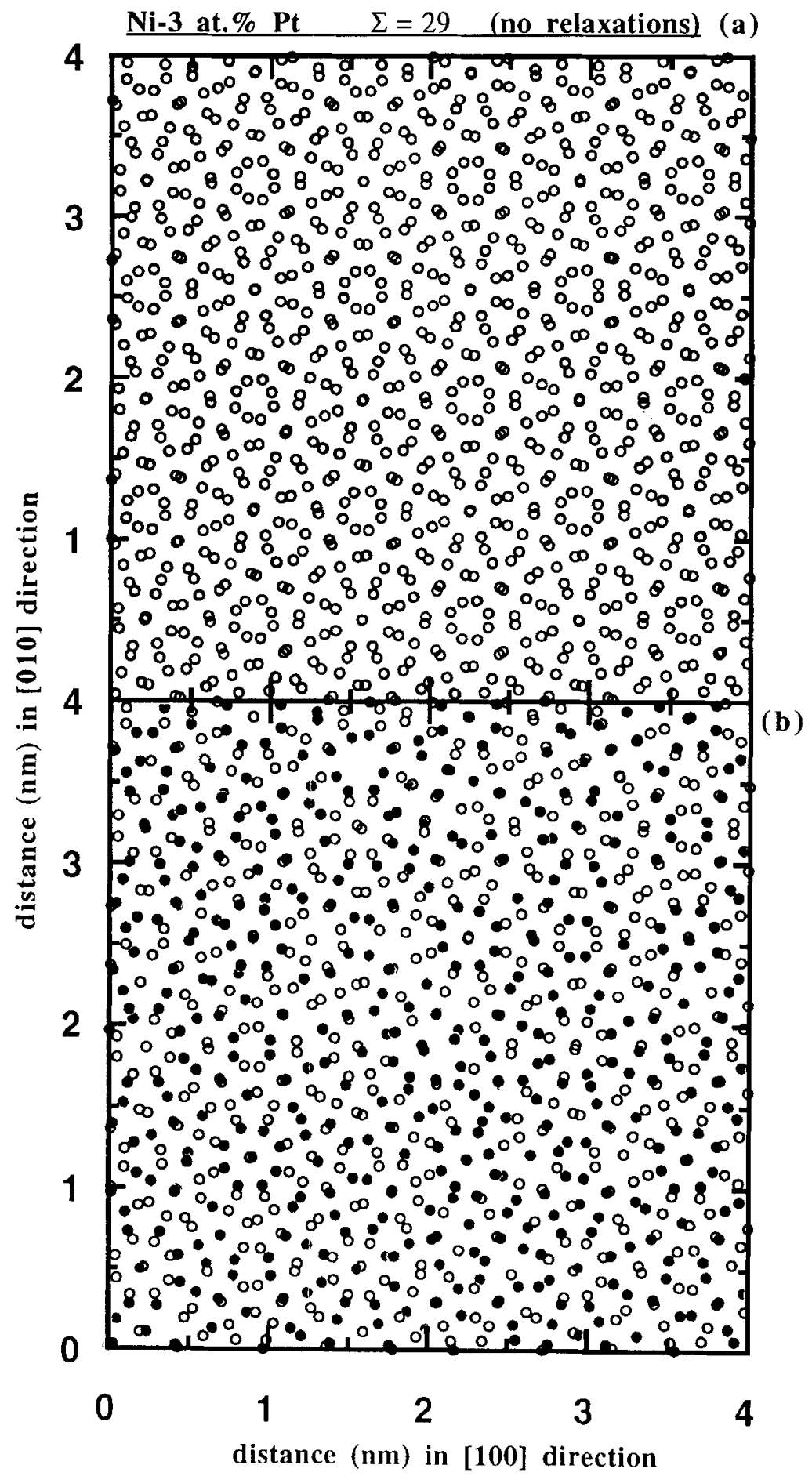

Fig. 14. The structure of and solute-atom segregation at the $\theta=43.6(\Sigma=29)$ boundary in Ni-3 at.\% Pt; same nomenclature as in Fig. 4 .

coincident sites to be enhanced in both the 1st and the 2nd planes on the Ni-rich side of the phase diagram. About $50 \%$ of the coincident sites, however, are not en- hanced in solute on the Pt-rich side, while many noncoincident sites are enhanced, especially in the 1st plane. Note also that Fig. 2 for the $\Sigma=5$ boundary clearly 
exhibits a break between very strong enhancement at some sites, and relatively low enhancement at the rest. By comparison with Fig. 10 the strongly enhanced sites are easily identified as coincident sites, constituting $20 \%$ of all the sites in the first plane adjacent to the interface. The sites with low solute enhancement are noncoincident sites.

3.2.5 $\Sigma=29\left(\theta=43.6^{\circ}\right)$. Comparing Figs. 13 and 14 we see that the relaxed GB structures are rather regular in both plots, and the segregation behavior seems to be complementary on both sides of the phase diagram. In accordance with the general trend the coincident sites tend to be enhanced in solute on the $\mathrm{Ni}$-rich side (Fig. 14), and not enhanced on the Pt-rich side (Fig. 13). It is difficult, however, in simple terms to account fully for the segregation patterns in this structure. It can be interpreted as a transition to the region of "general" boundaries. This is even clearer for the more general $\Sigma=73$ and $\Sigma=289$ GBs-not displayed here. They exhibit considerable structural disorder, although rather distorted regions of $\Sigma=5$ can often be identified. Finally, we emphasize that the enhancement levels are very high in all those boundaries.

\subsection{Correlation Between the Distance from the Interface and Solute Atom Concentration}

Despite the complexity of the structural dependence of segregation, there is one regularity that we have observed for all GBs. It has to do with the pattern of atomic relaxations in the (002) planes near the interface. It is well known from analytical models [58], computer simulations and experiments [32, 59], that local relaxations at (002) symmetrical twist boundaries lead to considerable variations in the positions of atomic sites not only in the plane parallel to the interface, but also in the direction normal to the interface within one (002) plane-up to $20 \%$ of the interplanar distance. Therefore, the relaxed structure of a (002) plane near the interface is not smooth, as in the bulk, but atomically rough, with different types of sites located closer to the interface or farther from it with respect to the average position of the plane normal to the interface. We now demonstrate for all the GBs discussed in the previous section that the regular pattern of atomic relaxation is clearly associated with solute-atom segregation on an atomic scale.

Figures 15 to 21 exhibit the dependence of the solute concentrations at atomic sites on the coordinate normal to the plane of the interface for two (002) planes each side of the interface. Part (a) of these figures refers to the Pt-rich side of the phase diagram and part (b) refers to the Ni-rich side. The geometrical plane of the interface is between the atomic planes -1 and 1 . Therefore, for the planes -2 and -1 the interface is to the right, and for the planes 1 and 2 it is to the left. The zero values on the abscissa correspond to the mean coordinate of the sites in a specific plane; only the portion $0.04 \mathrm{~nm}$ on each side of zero is exhibited-this region covers about $40 \%$ of the spacing between the (002) planes and contains all the atomic sites in each plane. Each point in the plot corresponds to one atomic site.

On the Ni-rich side the sites enhanced in solute tend to be closer to the interface in both the 1 st and 2 nd plane. On the Pt-rich side the dependence is the opposite, that is, the sites highly enhanced in solute tend to lie away from the interface. In both cases the effect is much stronger in the 1 st plane than in the 2 nd. Note also, that on the Pt-rich side at higher angles (Figs. 1821) a secondary branch of enhanced sites appears, that extends toward the interface. Figures 15 to 21 demonstrate, in detail, that the propensity for solute-atom segregation at individual GB sites is closely connected to the relaxation pattern of the sites with respect to the boundary plane-inward or outward. The scope and origin of this correlation is not clear at this point. A detailed comparison with other alloy systems and other GB geometries will be published elsewhere.

\section{Discussion and Summary}

We have investigated in detail regularities of soluteatom segregation at high-angle (002) symmetrical twist boundaries in dilute single-phase solid-solution alloys on both sides of the Ni-Pt phase diagram at $850 \mathrm{~K}$. The following is a discussion and summary of the results presented in Section 3.

\subsection{Effects of Chemistry}

The most general question with respect to solute-atom segregation is: how can we predict the segregating element? Often correlations are sought with the thermodynamic properties of the pure elements (surface tensions, cohesive energies, melting points) [60] or properties of the binary phase diagrams-solubility limits $[1,61]$ and slope of the liquidus [62]. From the point of view of elasticity theory-which has a reasonable track record in providing a rule of a thumb answer to different metallurgical problems [63]-the quantities of interest are the size and inhomogeneity misfit factors [63-70]. We limit our discussion to the 


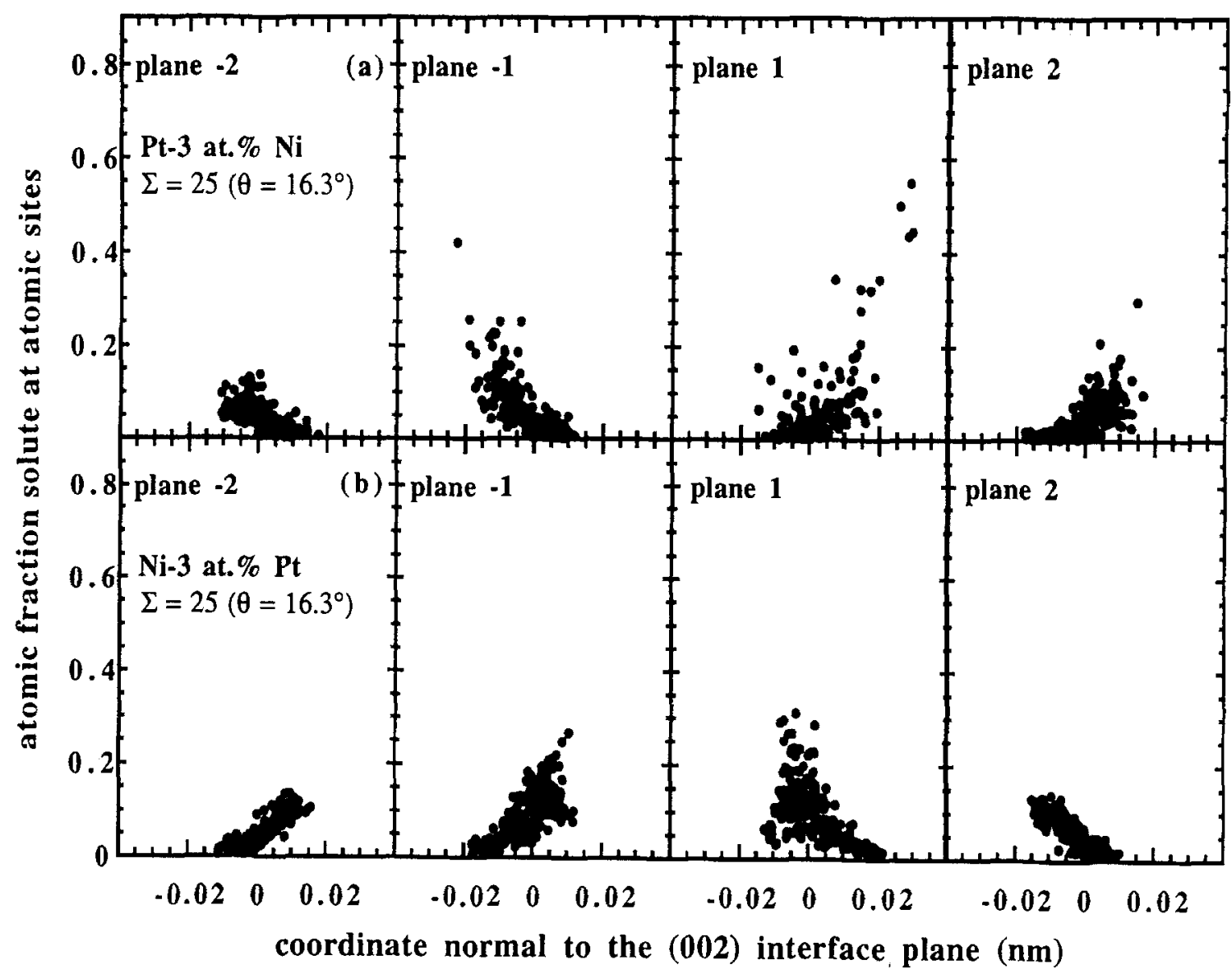

Fig. 15. The dependence of the solute concentration at atomic sites on the positions of the sites in the plane normal to the interface. Two (002) planes each side of the $\theta=16.3^{\circ}(\Sigma=25$ ) boundary are shown in: (a) Pt-3 at.\% Ni, and (b) Ni-3 at.\% Pt. Each atomic site is represented by a point. The abscissa of the point is the position of the site relative to the average position of the plane, and the vertical axis shows the mean concentration of solute at that site.

considerations of the size (or the lattice constant) of the solute and solvent atoms, bearing in mind that at least for the twist boundaries the inhomogeneity effect may be of no less importance [63-68]. The data are the results of computer simulations of solute-atom segregation at symmetrical twist (002) boundaries in f.c.c. metals at elevated temperatures utilizing the EAM potentials. The data on the $\mathrm{Ni}-\mathrm{Cu}$ and $\mathrm{Ag}-\mathrm{Au}$ system were obtained by Monte Carlo simulations [48, 71, 72] and free-energy minimization [41-44] methods. The data on $\mathrm{Au}-\mathrm{Pt}, \mathrm{Ni}-\mathrm{Pt}$ and $\mathrm{Au}-\mathrm{Ni}$ are from Monte Carlo simulations $[7-12,72]$, and the data on Au-Pd from freeenergy minimization [44] alone. The data are presented in Table 1 for both sides of each phase diagram in terms of the relative sizes of the solute and solvent atoms. The values for the size misfit factor $\left[\varepsilon_{a}=a^{-1}(d a / d c)\right]$ are taken from reference [73]. We now focus on the question as to whether solute or solvent is enhanced at the interface on each side of the phase diagram. There are only three mutually exclusive possibilities for each binary phase diagram: (a) the solute is enhanced on both sides; (b) the solvent is enhanced on both sides; and (c) the solute is enhanced on one side and the solvent is enhanced on the other. We now look for trends relating those possibilities and the magnitudes of the lattice constants of the solute and the solvent. Table 1 shows two approximate trends: ${ }^{2}$

1. The solute is enhanced on one side of the phase diagram and the solvent is enhanced on the other. Within this trend in all cases it is the larger lattice constant element that is enhanced at the GB.

2. The solute is enhanced at GBs on both sides of the phase diagram. ${ }^{3}$ This implies that on one side the smaller element is enhanced, and on the other side the larger element is enhanced. 


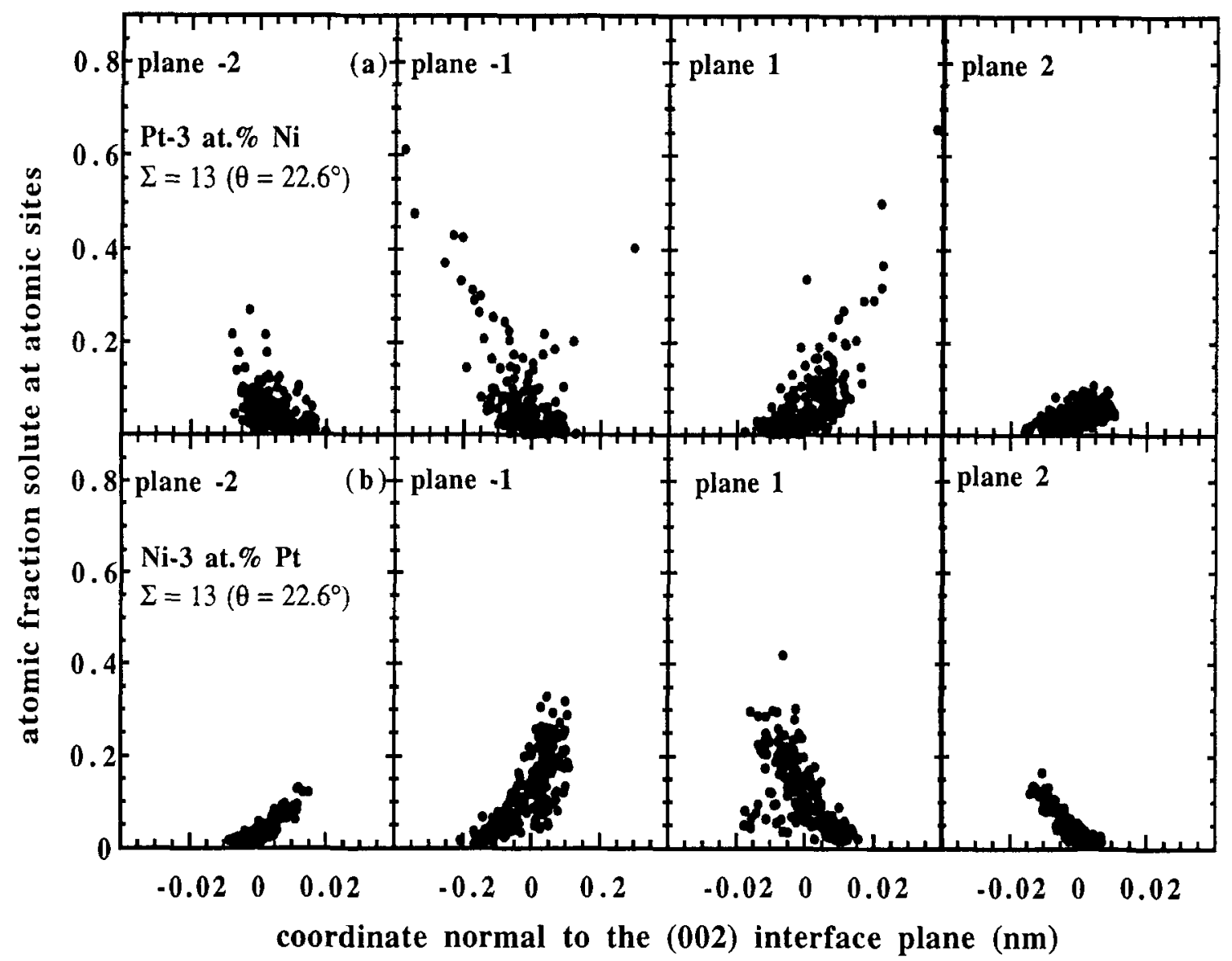

Fig. 16. The dependence of the solute concentration at atomic sites on the positions of the sites in the plane normal to the interface. Two (002) planes each side of the $\theta=22.6^{\circ}(\Sigma=13$ ) boundary are shown in: (a) Pt-3 at.\% Ni, and (b) Ni-3 at.\% Pt. Each atomic site is represented by a point. The abscissa of the point is the position of the site relative to the average position of the plane, and the vertical axis shows the mean concentration of solute at that site.

The first trend is compatible with the simple elasticity theory argument [64], that exchanging the solvent and the solute atoms in the dilute limit changes the signs of the solute-atom binding energies. ${ }^{4}$ This trend was studied in detail for low-angle boundaries in the Au-Pt system [7-10]. The sites enhanced in solute on the Pt-rich side form bipyramidal structures based on the square cells of the PGB screw dislocations. On the Au-rich side the same structures exhibited solute-atom depletion. The remainder of the sites are not affected by segregation in both cases.

The second trend was investigated in detail for the same low-angle GBs in the Ni-Pt system. The bipyramidal structures are enhanced in solute on the $\mathrm{Ni}$-rich side, while on the Pt-rich side they were unaffected by segregation-enhancement in solute was observed in the hourglass-like structures above the dislocation lines. At this point there is no clear explanation for this pattern. It requires a deeper understanding of detailed atomistic mechanisms of segregation.

\subsection{Distribution of Solute-Atom Site Concentrations Near Grain Boundaries}

The Ni-Pt system is the first system with a substantial enhancement of solute on both sides of the phase diagram to be studied by simulations. We calculated distributions of solute-atom concentrations for highangle boundaries in two (002) planes each side of the plane of the interface. For all the boundaries the enhancement levels are much smaller at the second plane, than in the first one, but they are still too strong to be disregarded. It is observed that the distribution is highly inhomogeneous in all cases-the ratio of solute concentrations between the sites with the highest and the lowest concentrations is between 25 to 30 . The 
Table 1. Regularities of solute-atom segregation from finite temperature simulations utilizing EAM potentials. The room temperature lattice constants of $\mathrm{Ag}, \mathrm{Au}, \mathrm{Cu}, \mathrm{Ni}, \mathrm{Pd}$ and $\mathrm{Pt}$ are correspondingly $0.4086,0.4079,0.3615,0.3517,0.3821$ and $0.3923 \mathrm{~nm}$. It should be borne in mind that the EAM potentials have been fitted to the dilute heats of solution only in the $\mathrm{Cu}-\mathrm{Ni}$ system.

\begin{tabular}{|c|c|c|c|c|c|}
\hline \multirow[b]{2}{*}{$\begin{array}{l}\text { Alloy } \\
\text { system }\end{array}$} & \multicolumn{2}{|c|}{$\begin{array}{c}\text { Small solvent atom-large } \\
\text { solute atom }\end{array}$} & \multicolumn{2}{|c|}{$\begin{array}{c}\text { Large solvent atom-small } \\
\text { solute atom } \\
\end{array}$} & \multirow[b]{2}{*}{ Reference } \\
\hline & $\begin{array}{l}\text { Segregating } \\
\text { element }\end{array}$ & $\begin{array}{l}\text { Size misfit } \\
\text { factor } \varepsilon_{a}\end{array}$ & $\begin{array}{l}\text { Segregating } \\
\text { element }\end{array}$ & $\begin{array}{l}\text { Size misfit } \\
\text { factor } \varepsilon_{a}\end{array}$ & \\
\hline $\mathrm{Au}-\mathrm{Ag}$ & Small $(A u)$ & -0.0021 & Large (Ag) & -0.0060 & {$[71,72]$} \\
\hline $\mathrm{Au}-\mathrm{Ni}$ & Small (Ni) & 0.1783 & Not available & -0.0792 & {$[72]$} \\
\hline $\mathrm{Au}-\mathrm{Pd}$ & Large $(\mathrm{Au})$ & 0.0510 & Large (Au) & -0.0498 & [44] \\
\hline $\mathrm{Au}-\mathrm{Pt}$ & Large $(\mathrm{Au})$ & 0.0352 & Large (Au) & -0.0440 & {$[7-11]$} \\
\hline $\mathrm{Ni}-\mathrm{Cu}$ & Large $(\mathrm{Cu})$ & 0.0233 & Large $(\mathrm{Cu})$ & -0.0290 & {$[48,71,72,41,44]$} \\
\hline $\mathrm{Ni}-\mathrm{Pt}$ & Large $(\mathrm{Pt})$ & 0.1337 & Small (Ni) & -0.0850 & {$[10,12]$} \\
\hline
\end{tabular}

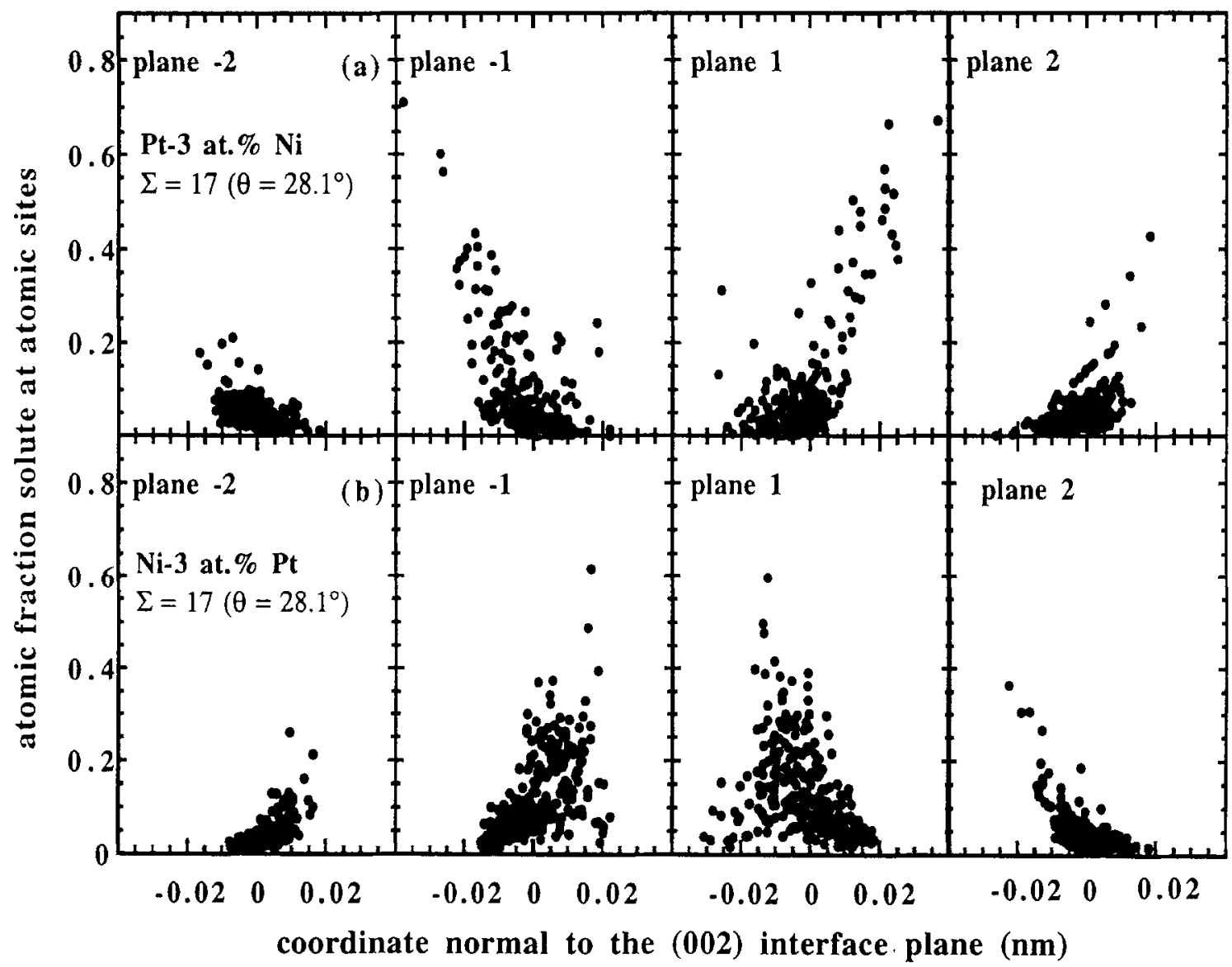

Fig. 17. The dependence of the solute concentration at atomic sites on the positions of the sites in the plane normal to the interface. Two (002) planes each side of the $\theta=28.1^{\circ}(\Sigma=17$ ) boundary are shown in: (a) Pt-3 at.\% Ni, and (b) Ni-3 at.\% Pt. Each atomic site is represented by a point. The abscissa of the point is the position of the site relative to the average position of the plane, and the vertical axis shows the mean concentration of solute at that site.

sites with the highest enhancement in solute are always in the minority, compared to the tail of sites with small or no enhancement [see Figs. (1) and (2)]. On the Pt-rich side of the phase diagram a small but statistically significant depletion in solute was observed at some sites. 


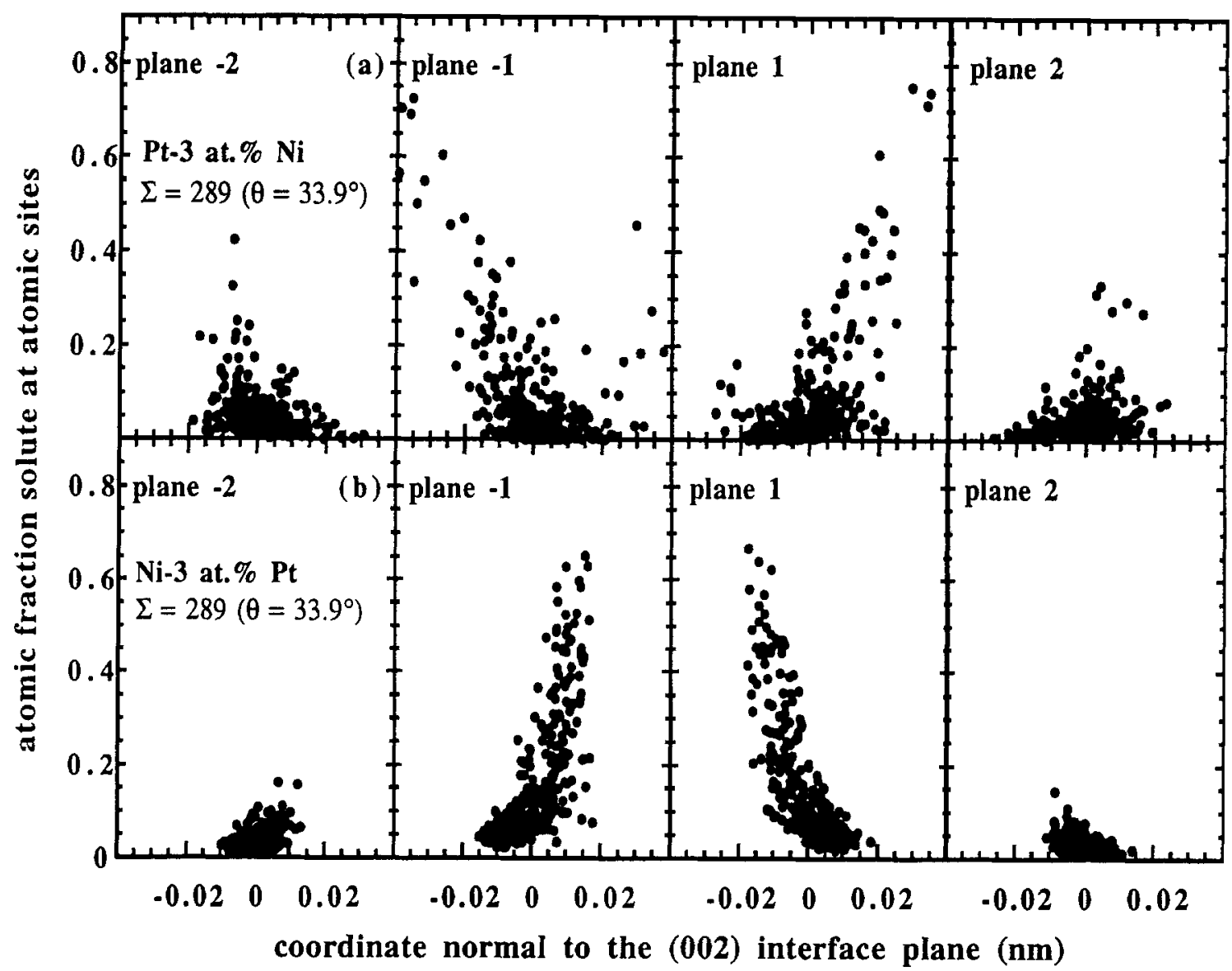

Fig. 18. The dependence of the solute concentration at atomic sites on the positions of the sites in the plane normal to the interface. Two (002) planes each side of the $\theta=33.9(\Sigma=289)$ boundary are shown in: (a) Pt-3 at.\% Ni, and (b) Ni-3 at.\% Pt. Each atomic site is represented by a point. The abscissa of the point is the position of the site relative to the average position of the plane, and the vertical axis shows the mean concentration of solute at that site.

The highest level of solute enhancement generally continues to increase even within the high-angle region up to values of close to $\Sigma=5$, where it saturates. These enhancement levels are much higher than the enhancement levels observed in low-angle boundaries, and are associated with cores of individual GB dislocations. It is demonstrated that the overlapping of the cores at high-angle GBs leads to a strong increase in solute-atom segregation at specific atomic sites.

Among the grain GBs investigated only the special $\Sigma=5$ boundary displays two clearly distinguishable segregation levels. All other boundaries exhibit fairly monotonic distributions. It is not surprising taking into account that the number of different symmetrically equivalent sites, $n$, in a twist boundary grows linearly with the value of $\Sigma$, that is, $n=1+(\Sigma-1) / 4$ [43]. Those sites have close values of segregation free energies and this leads to almost continuous distributions of solute-atom concentrations. This fact is very important for understanding the thermodynamics of $\mathrm{GB}$ segregation. In view of these results isotherms based on a single value of the segregation free energy appear to be unrealistic for most GBs; that is, a single value is only an effective free energy of segregation.

We have also shown that although high-angle GBs exhibit similar values of the integral quantity-the Gibbsian interfacial excess of solute-the distributions of solute concentrations at atomic sites near a boundary are different for all the boundaries investigated.

To have a frame of reference it is instructive to compare the present results on the distribution of solute-atom concentrations at the $(002)$ twist boundaries in Ni-Pt with the simulation results on other alloys. The best studied system is $\mathrm{Cu}-\mathrm{Ni}[42-44,48]$, 


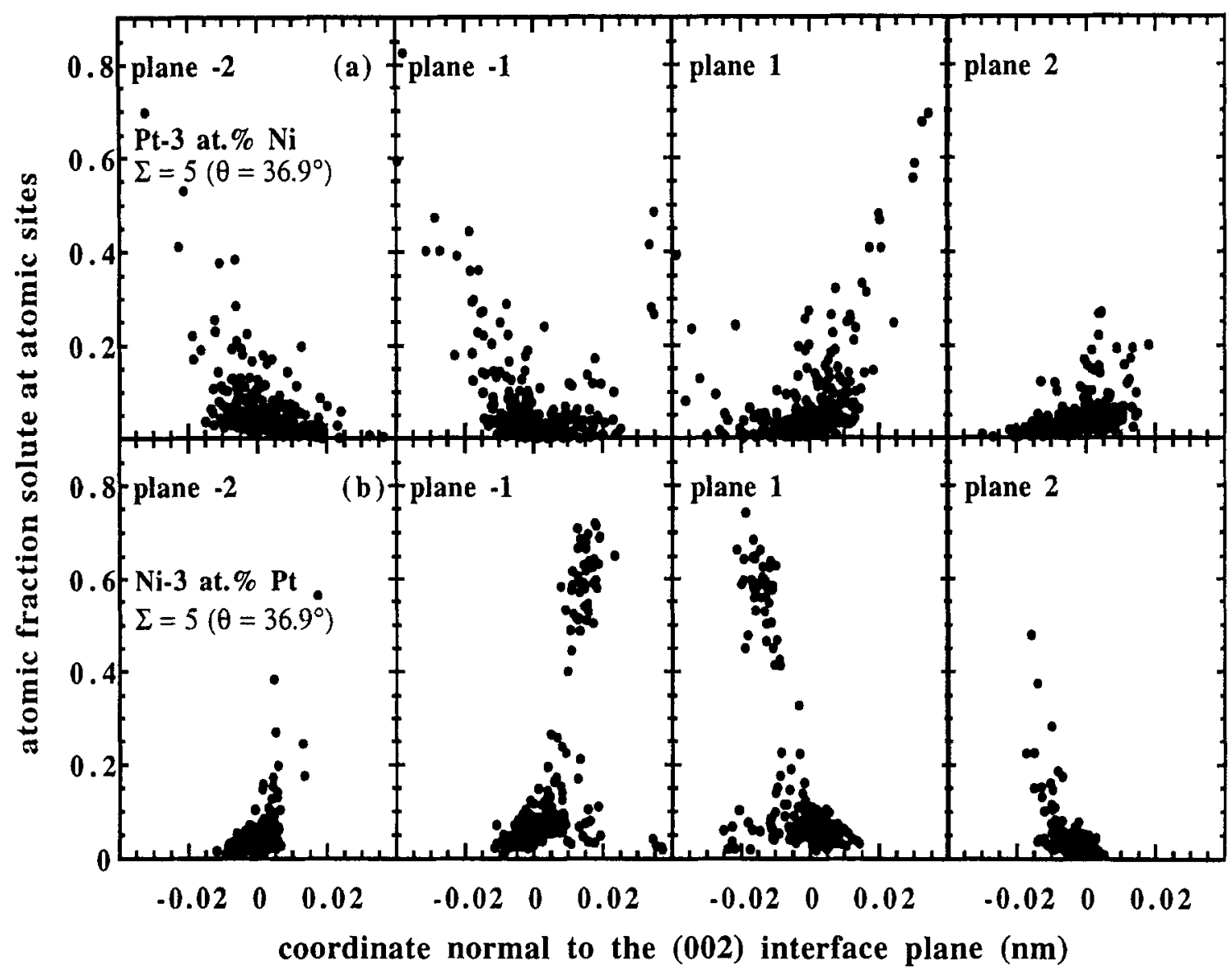

Fig. 19. The dependence of the solute concentration at atomic sites on the position of the sites in the plane normal to the interface. Two (002) planes each side of the $\theta=36.9(\Sigma=5)$ boundary are shown in: (a) Pt-3 at.\% Ni; and (b) Ni-3 at.\% Pt. Each atomic site is represented by a point. The abscissa of the point is the position of the site relative to the average position of the plane, and the vertical axis shows the mean concentration of solute at that site.

and the best studied GB is the $\Sigma=5 /(002)$. We now examine the Ni-rich sides of both phase diagrams, so that $\mathrm{Ni}$ is the solvent in both cases. Both solute species are larger than $\mathrm{Ni}$, but for $\mathrm{Cu}$ the difference is small (the size misfit factor is $\varepsilon_{a}=0.0233$ ), while for $\mathrm{Pt}$ it is about six time larger $\left(\varepsilon_{a}=0.1337\right)$. The data on the average solute concentration in the first plane adjacent to the interface in the $\mathrm{Cu}-\mathrm{Ni}$ system are compiled in Table 2 , which shows the results for the $\Sigma=5$ boundary at $T=800 \mathrm{~K}$ and for the bulk compositions, $c_{b}$ of 5 and 10 at. $\% \mathrm{Cu}$. The value in the 2 nd row was calculated from segregation binding energies for individual sites using the Langmuir-McLean relation (given in Table 3). The difference in the concentrations between the results of Monte Carlo simulations and free-energy minimization, as well as between different free-energy minimization studies is very large. The discrepancy between the free-energy minimization results is due to the different $\mathrm{Ni}$ - $\mathrm{Cu}$ EAM interatomic potentials used-those fitted to the dilute heats of solution [51], and the universal ones [52] which are not fitted to the dilute

Table 2. Cu concentrations in the first plane adjacent to the $\Sigma=$ $5 /(002) \mathrm{GB}$ in $\mathrm{Ni}(\mathrm{Cu})$ at $T=800 \mathrm{~K}$. The bulk concentrations of $\mathrm{Cu}$ are denoted $c_{b}$. The value in the second row is calculated from single atom segregation energies (see Table 3) using the LangmuirMcLean relation. Note well that the data from references [41, 43, 48] are obtained with the Ni-Cu potentials fitted to the dilute heats of solution [51]. While in references [42, 44] the universal potentials [52], not fitted to the heats of solution were used.

\begin{tabular}{lcl}
\hline$c_{b}=0.10$ at. fr. & $c_{b}=0.05$ at. fr. & Reference \\
\hline 0.740 & - & {$[48]$} \\
0.480 & - & {$[48]$} \\
0.856 & - & {$[41]$} \\
- & 0.680 & {$[43]$} \\
0.300 & 0.170 & {$[42,44]$} \\
\hline
\end{tabular}




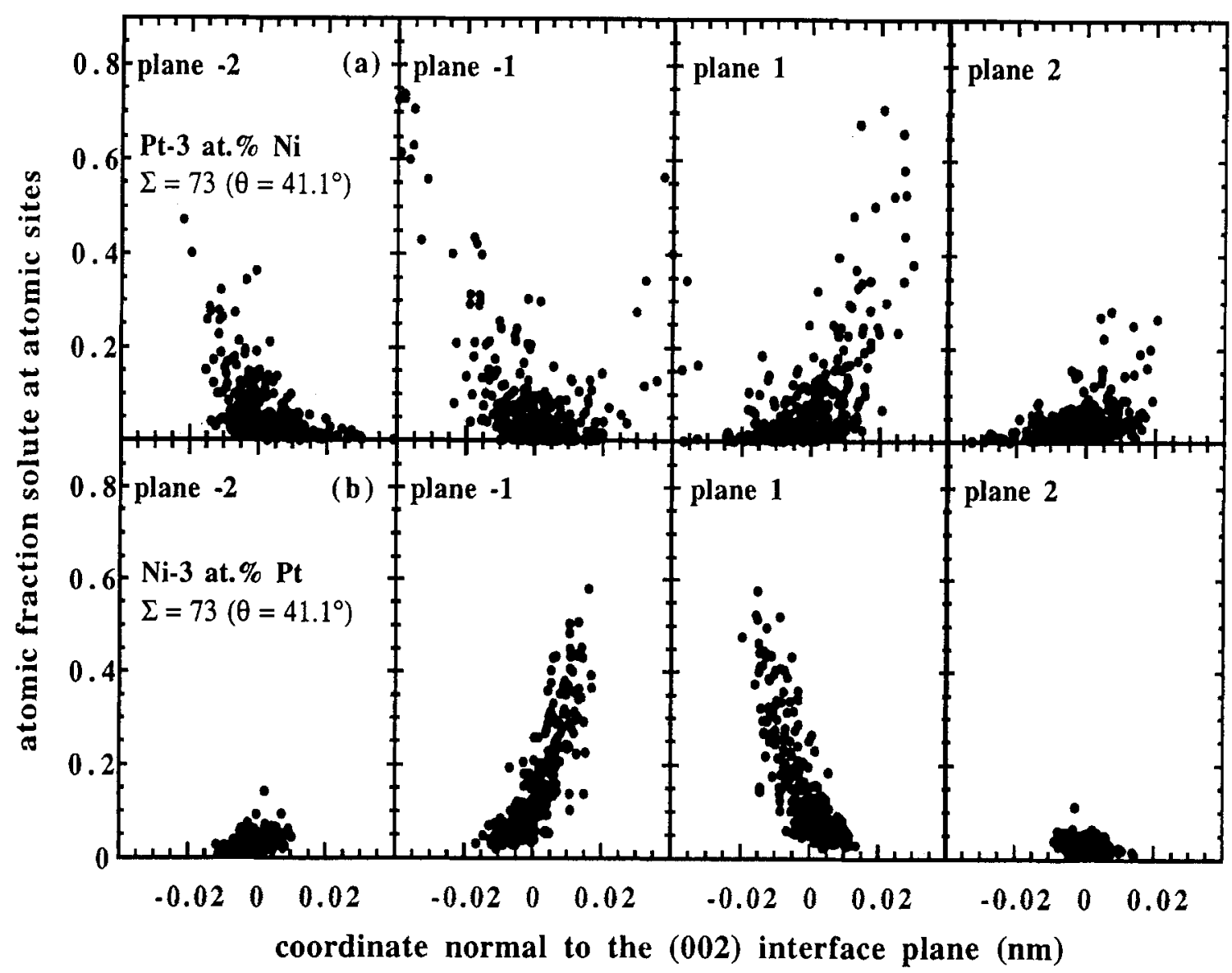

Fig. 20. The dependence of the solute concentration at atomic sites on the position of the sites in the plane normal to the interface. Two (002) planes each side of the $\theta=41.3^{\circ}(\Sigma=73$ ) boundary are shown in: (a) Pt-3 at.\% Ni; and (b) Ni-3 at.\% Pt. Each atomic site is represented by a point. The abscissa of the point is the position of the site relative to the average position of the plane, and the vertical axis shows the mean concentration of solute at that site.

Table 3. Segregation energies, $\Delta G$, and Cu concentrations for coincident and noncoincident sites in the first plane adjacent to the $\Sigma=5 /(002)$ $\mathrm{GB}$ in $\mathrm{Ni}(\mathrm{Cu})$ at $T=800 \mathrm{~K}$. The bulk concentrations of $\mathrm{Cu}$ are denoted $c_{b}$ (atomic fraction). The value of $\Delta G$ from reference [48] is the segregation energy at $0 \mathrm{~K}$, while in reference [44] it is the Helmholtz free energy of segregation. These values are used in the Langmuir-McLean relation to calculate the $\mathrm{Cu}$ concentrations at the corresponding sites, $c_{\text {calc }}$. The penultimate row shows the concentrations calculated in the free-energy minimization study [43]. Note well that the data from references $[43,48]$ are obtained with the Ni-Cu potentials fitted to the dilute heats of solution [51]. While in reference [44] the universal potentials [52], not fitted to the heats of solution, were used.

\begin{tabular}{lccccc}
\hline & $\begin{array}{c}\text { Coincident site, } c_{b} \\
0.05 \text { at. fr. }\end{array}$ & $\begin{array}{c}\text { Non-coincident site, } c_{b} \\
0.05 \text { at. fr. }\end{array}$ & $\begin{array}{c}\text { Coincident site, } c_{b} \\
0.10 \text { at. fr. }\end{array}$ & $\begin{array}{c}\text { Non-coincident site, } c_{b} \\
0.10 \text { at. fr. }\end{array}$ & Ref. \\
\hline$\Delta G(\mathrm{eV})$ & - & - & 0.220 & 0.130 & {$[48]$} \\
$c_{\text {zalc }}$ & - & - & 0.730 & 0.423 & {$[48]$} \\
$\Delta G(\mathrm{eV})$ & 0.115 & 0.082 & 0.118 & 0.090 & {$[44]$} \\
$c_{\mathrm{calc}}$ & 0.218 & 0.147 & 0.381 & 0.291 & {$[44]$} \\
$c_{\mathrm{obs}}$ & 0.800 & 0.650 & - & - & {$[43]$} \\
\hline
\end{tabular}

heats of solution. In all cases, however, the concentration of solute is of the same magnitude or even higher than in the Ni-Pt case. It clearly contradicts the idea that the magnitude of the segregation is larger in a system with a larger value of $\varepsilon_{a}$. Table 3 exhibits solute binding energies ${ }^{5}$ and solute concentrations separately for the 


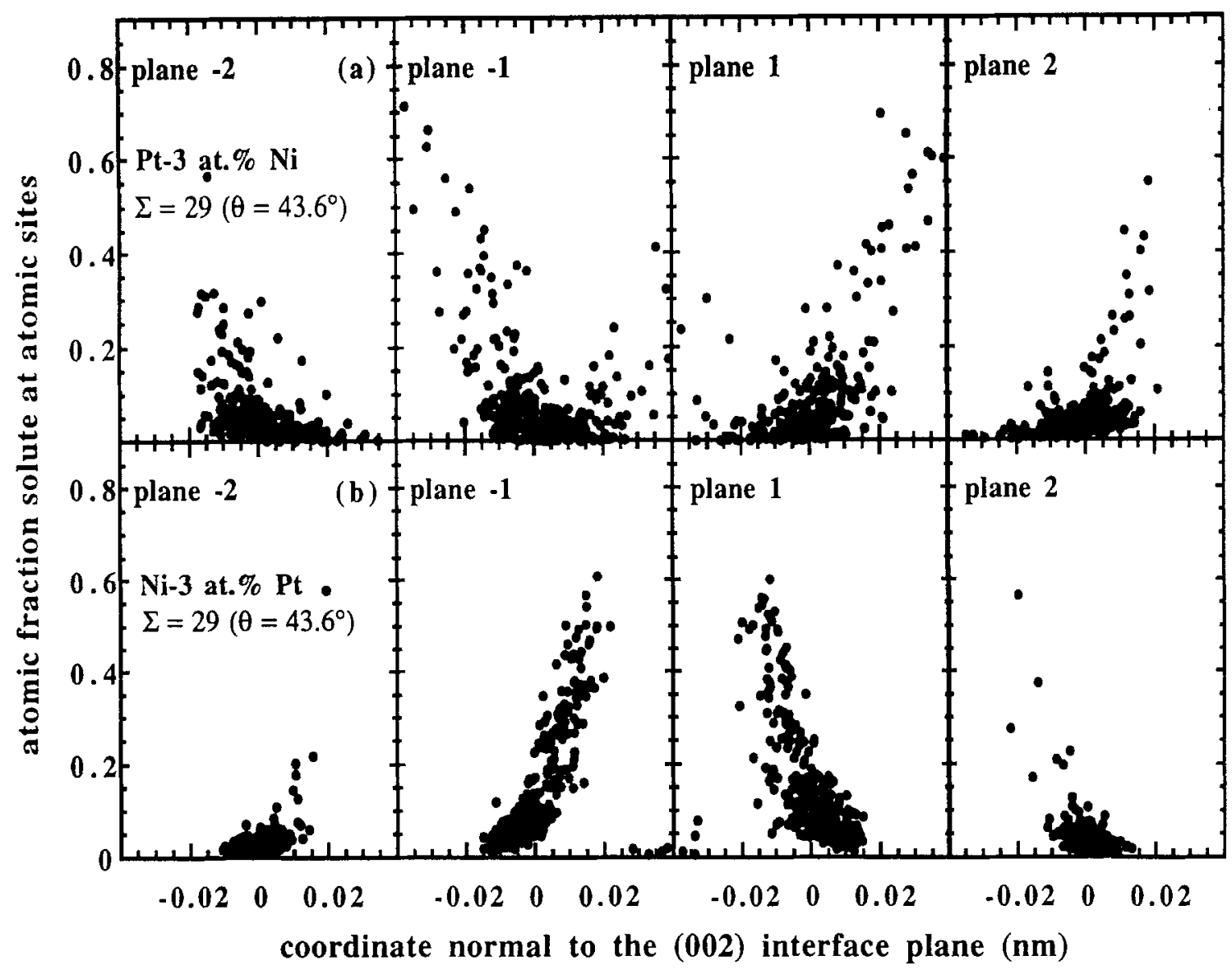

Fig. 21. The dependence of the solute concentration at atomic sites on the position of the sites in the plane normal to the interface. Two (002) planes each side of the $\theta=43.6^{\circ}(\Sigma=29$ ) boundary are shown in: (a) Pt-3 at.\% Ni; and (b) Ni-3 at.\% Pt. Each atomic site is represented by a point. The abscissa of the point is the position of the site relative to the average position of the plane, and the vertical axis shows the mean concentration of solute at that site.

two types of sites-coincident and non-coincident-in the first plane, and the binding free energies for the coincident and non-coincident sites obtained from Monte Carlo simulations [48] and free-energy minimization studies [44]. A positive sign of the binding energies implies enhancement of solute at a GB. In the dilute limit the Langmuir-McLean equation is expected to hold:

$$
\frac{c_{i}}{1-c_{i}}=\frac{c_{b}}{1-c_{b}} \exp \left(\frac{\Delta G_{i}}{k_{B} \Gamma}\right),
$$

where $c_{b}$ is the concentration of solute in the bulk, $c_{i}$ is the average concentration of solute at a boundary site of type $i$, and $\Delta G_{i}$ is the binding free energy to that site. Note well that this equation is applied to a particular type of $\mathrm{GB}$ site, not to all $G B$ sites, therefore no assumptions about the saturation value are necessary. The discrepancy between the results of Monte
Carlo simulations and the free-energy minimization is again considerable. In both cases, however, the site concentrations calculated from binding energies are much smaller than actually observed in the simulations. In reference [48] this effect is attributed to clustering tendencies in the $\mathrm{Cu}-\mathrm{Ni}$ system. It implies that atoms of the same species tend to cluster together, creating a concentration higher than the ensemble average. Note that in the $\mathrm{Cu}-\mathrm{Ni}$ system the ratio of the solute concentrations at the coincident and non-coincident sites is a factor of two. While in the Ni-Pt case, this ratio is more than an order of magnitude higher. Taking into account that the Ni-Pt system exhibits a clear tendency to chemical ordering, with three ordered phases $\left(\mathrm{NiPt}_{3}\right.$ and $\left.\mathrm{Ni}_{3} \mathrm{Pt}\right)$ in the bulk phase diagram [74], an ordered GB phase [75] may be responsible for the large concentration differences between different $\mathrm{GB}$ atomic sites in this system. Due to the spectrum of types of 
crystallographically equivalent sites at a GB the stoichiometry of the GB ordered phases may be different from those in the bulk phases.

The above results show that simple isotherms can fail even at relatively small bulk solute-concentrations and complete studies of the GB solutions throughout the phase diagram are necessary, as well as calculations of segregation free energies of a single solute atom at different GB sites. Finally, it appears, that although simple computational methods-like freeenergy minimization-often correctly reproduce some trends in segregation, extreme caution should be exercised when using this data for quantitative discussion. Recently problems have been detected in the values of single-atom segregation free-energies calculated within the local-harmonic approximation at elevated temperatures $[76,77]$. The discrepancies can be expected to be even larger for finite concentrations of solute due to the assumptions of the method. These results need to be verified by Monte Carlo and overlapping distributions Monte Carlo simulations.

\subsection{Atomistic Structure of Grain Boundaries and Segregation}

In our previous studies of low-angle boundaries [7-12] we found the spatial distribution of enhanced sites in the Ni-Pt system to be complementary on the two sides of the phase diagram. On the Pt-rich side the sites enhanced in solute form hourglass-like structures based on the primary GB screw dislocations. Alternatively, on the Ni-rich side the sites enhanced in solute form bipyramidal structures based on the cells of the same dislocation grid. The extent of the segregation regions and relatively low enhancement levels clearly point to the elastic origin of this effect, that is, the interaction between the solute atoms and the elastic stress fields of GBs. Similar effects were observed in our earlier investigations of low-angle GBs in the Au-Pt system [7-10], as well as in Monte Carlo simulations [48] and free-energy minimization [42] investigations of lowangle $\mathrm{GBs}$ in the $\mathrm{Cu}-\mathrm{Ni}$ system.

In this study we considered in detail the enhancement levels at different atomic sites at high-angle GBs. The trends observed are generally in agreement with those at low-angle GBs: $\mathrm{Ni}$ atoms tend to segregate to atomic sites that can be associated with the cores of PGBDs (the filler unit in terms of the structural unit model), while Pt atoms prefer sites at the patches of the f.c.c. structure $A$ units. The $B$ and $\beta$ units, that are associated with intersections of the primary screw dislocations, become important at high angles. Those regions are enhanced in solute on the $\mathrm{Ni}$-rich side of the phase diagram and are not enhanced on the Pt-rich side. There are, however, deviations from the general trend. For example, all coincident sites in the $\Sigma=5$ boundary on the Ni-rich side are strongly enhanced in solute, while the same sites in the $\Sigma=5$ boundary on the Pt-rich side are enhanced in approximately $50 \%$ of the cases. This and some other deviations from the general trends may be due to a certain amount of structural disorder at the GB structure, and/or chemical ordering effects. To understand it we need detailed information about the concentration dependence of segregation, and reliable values of the dilute free energies of segregation.

Another issue of considerable importance is the relation between the so-called special and general GBs. Traditionally, GBs with low $\Sigma$ values $(\Sigma<30)$ are called special. They exhibit a large degree of periodic matching and have relatively coarse displacement shift complete (DSC) lattices. Large deviations from these special misorientations lead to a decrease in the Burgers vectors of the corresponding SGBDs, as well as a decrease in the spacing between the SGBDs. Such situations, where periodic order may be lost, are referred to as general boundaries. Little is known about their exact structure. Most of the investigations have been performed employing special boundaries: they require smaller periodic computational cells in simulations and their structure is easier to interpret using $\mathrm{x}$-ray diffraction techniques. The physical properties of such general boundaries, however, are often significantly different from those of general boundariesthey exhibit cusps in the interfacial energy vs. misorientation plots [78-80], peculiarities with respect to diffusion, migration, etc. [81]. Sometimes, phase transitions between special and general GBs are discussed $[45,81]$. The conventional wisdom states that special boundaries should have a smaller propensity for segregation than general boundaries, because of better periodic matching [13]. Our results, however, show that there is no significant difference in the amount of solute segregation between the two types of boundaries.

In this paper we have detected a rather general correlation between the position of an atomic site in the direction normal to the interface-within one (002) plane-and the level of enhancement at this site (Figs. 15 to 21). First, it is shown that at high angles the (002) planes exhibit a considerable degree of relaxation in the direction normal to the interface, with the positions of certain sites deviating from the average position of the plane by up to $10 \%$ of the interplanar 
spacing; this value is in good agreement with both experiments [32] and calculations [58]. On the Ni-rich side the strongly enhanced sites tend to lie closer to the plane of the interface, while on the Pt-rich side they relax away from the interface. This correlation exists for both the first and the second planes, but it is much stronger at the first plane. A secondary weaker branch of enhanced sites, lying closer to the interface, is observed at higher-angle boundaries on the Pt-rich side starting at $\theta=33.9^{\circ}$. The origin of this effect is not clear. It may be that this effect is due to the relation between the local pressures at atomic sites and the way these sites are relaxed. Experimentally this effect may be investigated by x-ray diffraction, but deconvolution from reciprocal lattice space is not straightforward and requires a model. Atom-probe field-ion microscopy offers a good alternative, because it provides coordinates of atoms in the direct space, although the size of the sampled area should be large enough to provide adequate statistics [82-86].

\subsection{Correlation Between Local Pressures at Atomic Sites and Segregation}

The difference in the chemical composition at atomic sites near an interface is related to the differences in the local environment at these sites. To understand the mechanisms of solute-atom segregation it is desirable to characterize this environment by some simple quantity. Reasonable candidates for this role are components of the local stress tensor $\sigma$-specifically the hydrostatic pressure which is given by:

$$
p=(1 / 3)\left(\sigma_{11}+\sigma_{22}+\sigma_{33}\right)
$$

and the shear stress

$$
\tau=\sqrt{\sigma_{13}^{2}+\sigma_{23}^{2}}
$$

where direction 3 is normal to the plane of the interface.

Those quantities were used to identify cores of GB dislocations and other imperfections $[87,88]$. A direct correlation between the local hydrostatic pressure and chemical composition was observed in a tight-binding molecular dynamics study of Ag monolayers deposited on a $\mathrm{Cu}$ substrate $[89,90]$; the larger $\mathrm{Ag}$ atoms tend to occupy atomic sites under tension, while the smaller $\mathrm{Cu}$ atoms occupy compressed sites, forming a hexagonal grid.

Recently, the local stresses [Eqs. (4) and (5)] were calculated for (002) twist boundaries [43] using the free-energy minimization technique in the localharmonic approximation. The results show strong hydrostatic tension at coincident and quasi-coincident sites at the boundaries. The tensile stresses appear to cause enhancement of $\mathrm{Cu}$ in the $\mathrm{Ni}-5$ at. \% $\mathrm{Cu}$ alloy. No apparent correlation between the local chemical composition and the shear stresses was observed. Specific values of the stresses are, however, unavailable which does not allow for a quantitative discussion.

For a complete understanding of the stress-composition correlation it is important to know the distribution of stresses at atomic sites near an interface, how these values depend on the properties of the solvent (for example, the elastic moduli), and the response of chemical composition to the stresses in a reference bulk system. These issues are currently being addressed by Monte Carlo simulations.

\subsection{Structural Multiplicity Effects and Rigid-Body Translations in (002) GBs at Elevated Temperatures}

Current conventional wisdom about the structure of special GBs-derived from a substantial body of experiments and computer simulations-states that they have a uniform periodic structure up to the melting point. This structure is described in terms of SUs or, alternatively, in terms of PGBDs and SGBDs. In this paper we have shown that although this regularity is certainly strong and general, noticeable deviations can occur. We unambiguously observe a shifting of $\Sigma=5$ units by one atomic plane in the Ni-3 at.\% $\mathrm{Pt}$. This can be interpreted as roughening of the GB [91]. Experimental evidence of such roughening has recently been found in $x$-ray diffraction studies of $(002)$ twist GBs in gold [92]. A structural multiplicity is also observed in the nonshifted regions; it is difficult to clarify the origin of this phenomenon based on the present results. In other cases considerable instability is detected in the $\Sigma=5$ patches of the $\Sigma=13$ and $\Sigma=17$ boundaries on both sides of the phase diagram. In the case of $\Sigma=5$ on the Pt-rich side of the phase diagram the amount of disorder is considerable, and it is not straightforward to identify the alternative structures. We also observe certain rigidbody translations in some boundaries $-\Sigma=5$ and $\Sigma=13 \multimap$ on the Pt-rich side of the phase diagram. We emphasize that most of the studies of structures of GBs were performed by means of lattice statics at 0 $\mathrm{K}$. In this case a perfectly periodic structure is always expected to correspond to the free energy minimum. It 
was found, however, that even at $0 \mathrm{~K}$ the energy difference between structures with different symmetry is only $\sim 5 \%$ [31]. At elevated temperatures these alternative structures may appear or even prevail. A considerable fraction (about 10\%) of such structures was observed in molecular dynamics studies of the $\Sigma=5$ GB in gold at room temperature [35]. A study of thermodynamics of the same boundary by the free-energy minimization method in the local-harmonic approximation [39] suggests a first order-phase transition occurs around room temperature between two structures, which differ by a rigid-body translation in the plane of the interface. ${ }^{6}$ The microscopic mechanism of this transition is unknown. Should, however, it turn out to be a second order phase transition, then we may expect the system to exhibit premonitory effects over a wide temperature range. The increase of disorder in the structure, reported in this paper, may be a manifestation of such effects. Similar small, but statistically significant disordering was observed in a Monte Carlo simulation of the $\Sigma=13$ (002) twist boundary in the Au-Ni system (those effects were attributed to segregation of $\mathrm{Ni}$ ) [71]. Apart from the phase transition hypothesis, we can also imagine that GBs at elevated temperatures may host certain equilibrium structural defects, different from those in a three-dimensional crystal. Finally, the role of GB roughening is not yet clearly understood.

We are unaware of any conclusive experimental evidence, supporting the above speculations. At this point we cannot rule out the possibility that those phenomena are due to inadequacies of the existing potentials. If it turns out, however, that the effects discussed are of physical origin, they may have important implications for understanding different GB properties and phenomena, including diffusion and migration.

The presence of all these effects emphasizes the need for Monte Carlo and molecular dynamics simulations of interfacial phenomena at elevated temperatures. Those methods are universal, because they perform a thermodynamically rigorous sampling of configurations at equilibrium, independent of the particular structure of the configuration space. Furthermore, those methods, given adequate statistics cannot miss any significant contributions from possible imperfections or alternative structures. The resulting averages, therefore, provide a reliable picture of the phenomena. The free energy minimization techniques, on the other hand, seek the minimum of the free energy and work well when this minimum is deep enough. In situations of structural multiplicity or partial disordering the free energy surface in the multidimensional phase space of GB variables may be relatively flat, and the system may get stuck in one of many local minima. Similar considerations apply to the common practice of quenching Monte Carlo snapshots by lattice statics in order to obtain equilibrium structures. This technique, aimed at removing the noise due to thermal vibrations, may lead to a loss of important information about structural multiplicity.

\section{Conclusions}

1. We present results of Monte Carlo simulations of solute-atom segregation at high-angle symmetrical (002) twist boundaries for dilute single-phase alloys on both sides of the Ni-Pt phase diagram at $850 \mathrm{~K}$. The boundaries studied have the following $\theta$ misorientations: $16.3^{\circ}(\Sigma=25), 22.6^{\circ}(\Sigma=13)$, $28.1^{\circ}(\Sigma=17), 33.9^{\circ}(\Sigma=289), 36.9^{\circ}(\Sigma=5)$, $41.1^{\circ}(\Sigma=73)$ and $43.6^{\circ}(\Sigma=29)$. Solute-atom enhancement in this alloy occurs on both sides of the phase diagram, although it is considerably higher on the Ni-rich side.

2. Enhancement in solute is observed in the first two planes on both sides of the interface. Enhancement in the first plane is always much stronger than in the second plane. The distribution of solute concentrations within one plane is always very inhomogeneous with the highly enhanced-sites being in the minority. The remainder of the sites exhibit little or no enhancement. In some cases on the Pt-rich side a slight depletion in solute is observed for a small fraction of the sites.

3. The highest level of solute enhancement continues to increase up to the $\Sigma=5$ misorientation, where saturation occurs; the values of solute concentrations are about 25 times the bulk concentration. This large difference, compared to the situation in the Cu-Ni system, suggests the possibility of a chemically ordered $\mathrm{GB}$ phase.

4. Only the $\Sigma=5$ boundary, on the Ni-rich side, displays a clear separation of enhancement levels for the two possible types of sites. In most cases the distributions of solute concentrations are rather smooth, indicating a considerable overlapping of the enhancement levels at different types of sites.

5. Although the boundaries with misorientations close to $\Sigma=5$, and higher, exhibit similar enhancement 
levels the distributions of solute enhancements are quite different for all of them.

6. The correlation between the structure and solute concentration at the GB continues the trends observed for low-angle GBs [7-12]. The structural units associated with the perfect crystal are enhanced in solute on the Ni-rich side of the phase diagram, while on the Pt-rich side solute atoms prefer dislocation-like filler units. In the $\Sigma=5$ structural units on the Ni-rich side of the phase diagram the coincident sites are strongly enhanced in solute, while on the Pt-rich side there is no clear trend for these structural units.

7. Contrary to the conventional wisdom no significant difference in the maximum levels of segregation is observed between special and general GBs.

8. A general correlation is detected between the positions of sites in the first two planes with respect to the plane of the interface and the solute concentration. On the Ni-rich side the enhanced sites tend to lie closer to the interface, while on the Pt-rich side they tend to lie farther away from the interface. A secondary weaker branch of enhanced sites, lying closest to the interface, is observed at the highestangle boundaries on the Pt-rich side.

9. Rigid-body translations from the CSL positions are observed for the $\Sigma=5$ and $\Sigma=13 \mathrm{GBs}$ on the Pt-rich side of the phase diagram. GB roughening is observed in the $\Sigma=5$ boundary on the Ni-rich side. Possible structural multiplicity is detected in this boundary, as well as in the $\Sigma=5$ patches of some other boundaries. Significant disordering is observed for the $\Sigma=5$ boundary on the Pt-rich side.

\section{Acknowledgments}

This research is supported by the National Science Foundation (grant No. DMR-9319074, Dr. B. McDonald, grant officer). This work is also partially supported by grant number DMR920002N, and it utilizes the CRAY Y-MP and CRAY 2 systems at the National Center for Supercomputing Applications at the University of Illinois at Urbana-Champaign. This work made use of MRL Central facilities supported by the National Science Foundation, at the Materials Research Center of Northwestern University, under award No. DMR9120521. We wish to thank Drs. G. Martin and B. Legrand and Mr. J.D. Rittner for stimulating discussions.
Notes

1. Structural multiplicity is the occurrence of distinctly different structural units in the same GB environment. It implies that these alternative units have similar free energy values.

2. We do not consider the case of the Au-Ag alloy, because the magnitude of the effect is much smaller than in all other cases and there is a discrepancy between the results of Monte Carlo simulations [72] and free energy minimization [44] on the Aurich side, and, finally, the system is peculiar with respect to the Vegard's law -note the negative sign of the size misfit factor on both sides of the phase diagram.

3. Experimentally this pattern was observed in the Au-Pt system for an unspecified boundary (see references [93, 94]).

4. There exists a different phenomenological description of interfacial segregation, incorporating elastic effects [95].

5. The Gibbs binding free energy of a solute-atom to a particular boundary site $i, \Delta G_{i}$, is defined as the change in the free energy of a bicrystal due to exchanging one solute atom between a bulk site and a site $i$ at a boundary. The results taken from [44] are Helmholtz binding free energies, while [48] gives binding energies from lattice statics calculations at $0 \mathrm{~K}$, that are internal energy differences.

6. Studies of the same boundary by the same method in Ag-Au, Au-Pd and $\mathrm{Cu}-\mathrm{Ni}$ alloys $[43,44]$ over a wide range of temperatures and bulk compositions did not show the presence of the type I structure. This poses an important question about the possible relation between solute-atom segregation and $G B$ phase transitions.

\section{References}

1. M.P. Seah and E.D. Hondros, Proc. Roy. Soc. A 355, 191 (1975).

2. E.D. Hondros and M.P. Seah, in Physical Metallurgy, edited by R.W. Cahn and P. Haasen (North Holland, Amsterdam, 1983), p. 856 .

3. C.L. Briant, in Materials Interfaces: Atomic-Level Structure and Properties, edited by D. Wolf and S. Yip (Chapman \& Hall, London, 1992), p. 463.

4. S.M. Foiles and D.N. Seidman, in Materials Interfaces: AtomicLevel Structure and Properties, edited by D.Wolf and S.Yip (Chapman \& Hall, London, 1992), p. 497.

5. R. Kirchheim, in Materials Interfaces: Atomic-Level Structure and Properties, edited by D. Wolf and S. Yip (Chapman \& Hall, London, 1992), p. 481

6. J.W. Cahn, J. Phys. (Paris) 43, C6-192 (1982).

7. A. Seki, D.N. Seidman, Y. Oh, and S.M. Foiles, Acta Metall. Mater. 39, 3167, 3179 (1991).

8. D. Udler and D.N. Seidman, Phys. Status Solidi (b) 172, 267 (1992).

9. D. Udler and D.N. Seidman, Mater. Res. Soc. Symp, Proc. 278, 223 (1992).

10. D. Udler and D.N. Seidman, Materials Science Forum 126-128, 165 (1993).

11. D. Udler and D.N. Seidman, Materials Science Forum 126-128, 169 (1993).

12. D. Udler and D.N. Seidman, Acta Metall. Mater. 42, 1959 (1994). 
13. R.W. Balluffi, in Interfacial Segregation, edited by W.C. Johnson and J.M. Blakely (ASM, Metals Park, Ohio, 1979), p. 193.

14. D. McLean, Grain Boundaries in Metals (Clarendon Press, Oxford, 1957).

15. E.D. Hondros and M.P. Seah, Metall. Trans. 8A, 1363 (1977).

16. M. Guttmann, Metall. Trans. 8A, 1383 (1977).

17. C.L. White and W.A. Coghlan, Metall. Trans. 8A, 1403 (1977).

18. T. Mütschelle and R. Kirchheim, Scripta Metall. 21, 21135 (1987).

19. K. Lücke and K. Detert, Acta Metall. 5, 628 (1957).

20. J.W. Cahn, Acta Metall. 10, 789 (1962).

21. K. Lücke and H.P. Stüwe, Acta Metall. 19, 1087 (1971).

22. D.A. Molodov, V.Ye. Fradkov, L.S. Shvindlerman, and G.I. Kaplan, Sov. Phys. Solid State 26, 284 (1984).

23. R.G. Hoagland, M.I. Baskes, M.S. Daw, and S.M. Foiles, J. Mater. Res. 5, 313 (1990).

24. M.I. Baskes, R.G. Hoagland, and A. Needleman, Mater. Sci. Eng. A 159, 1 (1993).

25. W.T. Read, Dislocations in Crystals (McGraw Hill, New York, 1953).

26. J.C.M. Li, in Electron Microscopy and Strength of Crystals, edited by G. Thomas and J. Washburn (John Wiley, New York, 1963), p. 713.

27. D.N. Seidman, in Materials Interfaces: Atomic-Level Structure and Properties, edited by D. Wolf and S. Yip (Chapman \& Hall, London, 1992), p. 58.

28. T. Schober and R.W. Balluffi, Philos. Mag. 21, 109 (1970).

29. D. Schwartz, V. Vitek, and A.P. Sutton, Philos. Mag. A 51, 499 (1985).

30. D. Schwartz, V. Vitek, and P.D. Bristowe, Acta Metall. 36, 675 (1988).

31. P.D. Bristowe and A.G. Crocker, Philos. Mag. A 38, 487(1978).

32. M.S. Taylor, I. Majid, P.D. Bristowe, and R.W. Balluff, Phys. Rev. B 40, 2772 (1989); I. Majid, P.D. Bristowe, and R.W. Balluffi, Phys. Rev. B 40, 2779 (1989),

33. D. Wolf, Acta Metall. 32, 245, 735 (1984); Physica 131B, 53 (1985); in Materials Interfaces: Atomic-Level Structure and Properties, edited by D. Wolf and S. Yip (Chapman \& Hall, London, 1992), p. 1.

34. Y. Oh and V. Vitek, Acta Metall. 34, 1941 (1986).

35. I. Majid and P.D. Bristowe, Scripta Metall. 21, 1153 (1987).

36. J.F. Lutsko, D. Wolf, S. Yip, S.R. Phillpot, and T. Nguyen, Phys. Rev. B 38, 11572 (1988).

37. S.M. Foiles, Acta Metall. 37, 2815 (1989).

38. G.A. Evangelakis and V. Pontikis, Europhysics Lett. 8, 599 (1989).

39. R. Najafabadi, D.J. Srolovitz, and R. LeSar, J. Mater. Res. 5, 2663 (1990).

40. R. Najafabadi, D.J. Srolovitz, and R. LeSar, J. Mater. Res. 6, 999 (1991).

41. R. Najafabadi, D.J. Srolovitz, H.Y. Wang, and R. LeSar, Acta Metall. Mater. 39, 3071 (1991).

42. H.Y. Wang, R. Najafabadi, and D.J. Srolovitz, Philos. Mag. A 65, 625 (1992).

43. H.Y. Wang, R. Najafabadi, D.J. Srolovitz, and R. LeSar, Acta Metall. Mater. 41, 2553 (1993).

44. H.Y. Wang, R. Najafabadi, D.J. Srolovitz, and R. LeSar, Interface Science 1, 31 (1993).

45. C. Rottman, J. Phys. (Paris) 43, C5-313 (1988).

46. J. Rittner, D. Udler, and D.N. Seidman, Phys. Rev. Lett. 74, 1115 (1995).
47. S.M. Foiles, in Surface Segregation and Related Phenomena, edited by P.A. Dowben and A. Miller (CRC Press, Boca Raton, Florida, 1990), p. 79.

48. S.M. Foiles, Phys. Rev. B 40, 11502 (1989).

49. N. Metropolis, M.N. Rosenbluth, A.W. Rosenbluth, A.H. Teller, and E. Teller, J. Chem. Phys. 21, 1087 (1953).

50. M.S. Daw and M.I. Baskes, Phys. Rev. Lett. 50, 1285 (1983); Phys. Rev. B 29, 6443 (1984).

51. S.M. Foiles, Phys. Rev. B 32, 7685 (1985).

52. S.M. Foiles, M.I. Baskes, and M.S. Daw, Phys. Rev. B 33, 7983 (1986); Phys. Rev. B 37, 10378 (1988).

53. M.S. Daw, Phys. Rev. B 39, 7441 (1989).

54. M.S. Daw, S.M. Foiles, and M.I. Baskes, Mater. Sci. Repts. 9, 251 (1993).

55. R.C. Pond and V. Vitek, Proc. Roy. Soc. Lond. B 357, 453 (1977).

56. A.M. Papon, M. Petit, G. Silvestre and J.J. Bachmann, Mat. Res. Soc. Symp. Proc. 5, 27 (1982).

57. K.L. Merkle and D.J. Smith, Phys. Rev. Lett. 59, 2887 (1987).

58. J.P. Hirth and B. Carnahan, Acta Metall. 40, 1237 (1992).

59. M.R. Fitzsimmons and S.L. Sass, Acta Metall. 36, 3103 (1988); Acta Metall. 37, 1009 (1989).

60. P. Wynblatt and R.C. Kuo, in Interfacial Segregation, edited by W.C. Johnson and J.M. Blakely (ASM, Metals Park, Ohio, 1979), p. 115.

61. S. Hofmann and P. Lejcek, Scripta Metall. 25, 2259 (1989).

62. J.J. Burton and E.S. Machlin, Phys. Rev. Lett. 37, 1433 (1981).

63. J.D. Eshelby, in Solid State Physics (Academic, New York, 1956), v. 3, p. 79.

64. D. Udler and D.N. Seidman, Scripta Metall. Mater. 26, 449, 803 (1992).

65. H. Stehle and A. Seeger, Z. Phys. 146, 217 (1956); A. Seeger and P. Haasen, Philos. Mag. 3, 470 (1958).

66. R.L. Fleischer, Acta Metall. 11, 203 (1961).

67. I. Saxl, Czech. J. Phys. B 14, 381 (1964).

68. B. Smola, Czech. J. Phys. B 31, 447 (1981).

69. L.A. Gypen and Deruyterre, Scripta Metall. 15, 815 (1981).

70. O. Boser, J. Appl. Phys. 44, 1038 (1973).

71. P.D. Bristowe, I. Majid, C. Counterman, D. Wang, and R.W. Balluffi, Materials Science Forum 126-128, 25 (1993),

72. C.A. Counterman, Ph.D. thesis, MIT (1991).

73. H.W. King, J. Mater. Sci. 1, 79 (1966).

74. M. Hansen and K. Anderko, Constitution of Binary Alloys (McGraw Hill, New York, 1958); C.E. Dahmani, M.S. Cadeville, J.M. Sanchez and J.L. Moran-Lopez, Phys. Rev. Lett. 55, 1208 (1985).

75. R. Herschitz and D.N. Seidman, Acta Metall. 33, 1547, 1576 (1985).

76. S.M. Foiles, Phys. Rev. B 49, 14930 (1994).

77. J.D. Rittner, S.M. Foiles, and D.N. Seidman, Phys. Rev. B 50, 12004 (1994).

78. A. Brokman and R.W. Balluffi, Acta Metall. 29, 1703 (1981).

79. R.W. Balluffi and A.P. Sutton, Acta Metall. 35, 2177 (1987).

80. D. Wolf, Acta Metall. 37, 1983, 2823 (1989); Acta Metall. 38, 781, 791 (1990).

81. L.S. Shvindlerman and B.S. Straumal, Acta Metall. 33, 1735 (1985).

82. S.-M. Kuo, A. Seki, Y. Oh, and D.N. Seidman, Phys. Rev. Lett. 65, 199 (1990).

83. J.G. Hu and D.N. Seidman, Phys. Rev. Lett. 65, 1615 (1990).

84. J.G. Hu and D.N. Seidman, Scripta. Metall. Mater. 26, 633 (1992). 
85. B.W. Krakauer and D.N. Seidman, Phys. Rev. B 65, 6724 (1993).

86. D.N. Seidman, B.W. Krakauer, and D.K. Chan, MSA Bullet. 24(1), 375 (1994); D.N. Seidman, B.W. Krakauer, and D. Udler, J. Phys. Chem. Solids, 55, 1035 (1994).

87. T. Egami, K. Maeda, and V. Vitek, Philos. Mag. 41, 883 (1980).

88. A.P. Sutton and V. Vitek, Phil. Trans. R. Soc. Lond. A309, 1 (1983).

89. G. Tréglia, B. Legrand, J. Eugène, B. Aufray, and F. Cabané, Phys. Rev. B 44, 6842 (1991).

90. C. Mottet, G. Tréglia, and B. Legrand, Phys. Rev. B 46, 16018
(1992); Surf. Sci. 287/288, 476 (1993).

91. F. Schmid and K. Binder, Phys. Rev. B 46, 13533, 13565 (1992).

92. M.R. Fitzsimmons and E. Burkel, Phys. Rev. B 47, 8436 (1993).

93. J.E. Hilliard, M. Cohen, and B.L. Averbach, Acta Metall. 8, 26 (1960).

94. B.S. Bokshteyn, L.M. Klinger, G.S. Nikolsky, V.Ye. Fradkov, and L.S. Shvindlerman, Phys. Metals. Metallogr. 48, 75 (1981).

95. F.F. Abraham, N.H. Tsai, and G.M. Pound, Scripta Metall. 13,307 (1979); F.F. Abraham, Phys. Rev. Lett. 46, 546 (1981); F.F. Abraham and C.R. Brundle, J. Vac. Sci. Technol. 18, 506 (1981). 